\title{
Monte-Carlo Simulation of Compatibilization by Network-Building and Catalytic Interface Reactions in Two-Component Injection Molding
}

\author{
Andreas John*, Jürgen Nagel and Gert Heinrich
}

Leibniz Institut für Polymerforschung Dresden e. V., Hohe Strasse 6, 01069 Dresden, Germany

\begin{abstract}
Adhesion of immiscible polymers during two-component injection molding can be improved by transreactions of properly functionalized molecules in situ by exploitation of the thermal energy of the melts. These reactions must provide a sufficient conversion of reactive monomers in the short cooling time down to the glass temperature. Furthermore, as much as possible interconnecting chemical links on the molecular level have to be created between the components within the small spatial region of the interdiffusion interface width. To investigate these processes, we performed MonteCarlo (MC) simulations based on the three-dimensional coarse-grained Bond Fluctuation Model (BFM) including a thermal interaction potential in $r \leq \sqrt{6}$ with energy $\varepsilon=0.1 k_{B} T$. We compared a simple Split type reaction, which is capable of network-forming, with a catalytic interface reactive process both exhibiting different values of activation energy. The main process of the catalytic reaction system is identical to the simple Split reaction as described in a previous paper, but now a reactive monomer creating process is prefixed. For the reacting systems different physical properties like consumption, radius of gyration, concentration profiles or the distribution of the degree of polymerization were calculated as a function of time. Additionally, several functions for the description of the adhesive strength on the molecular level were adopted and calculated depending on reaction type, activation energy and degree of consumption, respectively. From the results, those chemical reaction types were deduced, which should be most suitable for compatibilization intentions in two-component injection molding.
\end{abstract}

Keywords: Injection molding, reactive compatibilization, monte-carlo simulation.

\section{INTRODUCTION}

Two-component injection molding is a widely used technology in polymer processing industries to combine characteristic properties (e.g., thermal stability, elasticity, permeability) of different polymers within one final product. In this way, it is possible to improve the performance of a composite and to take advantage of the most favourable properties of both the materials [1].

Unfortunately, the miscibility of most polymers is very low, and the strength of the interface between the two materials is only determined (i) by Van der Waals interactions between the polymer molecules and (ii) by entropic forces allowing chains being located within the interpenetration depth crossing the interface. Physical entanglements, which will improve the adhesion, can only be performed in the short time of cooling down to the glass temperature and within the very small region of interdiffusion of few nanometers [2], and are only little pronounced.

But in many cases physical adhesion alone does not lead to the performance properties for everyday use. However, an improvement of adhesion of different polymers may be expected generally by (i) flow induced acceleration of the reactive coupling rate in melt mixers [3], (ii) deposition of diblock copolymers of both of the components inside the interdiffusion boundary layer [4-6] or (iii) in situ formation of chemical bonds between the two components during a

*Address correspondence to this author at the Leibniz Institut für Polymerforschung Dresden e. V., Hohe Strasse 6, 01069 Dresden, Germany; Tel: +49 3514658 751; Fax: +49 3514658 752; E-mail: john@ipfdd.de reactive extrusion process [7-9]. This latter favourable technique may also be successfully extended to the injection molding procedure and in this way take advantage of the thermal energy of the melts [10]. But in the short cooling time down to the glass temperature of only few seconds and the small spatial dimensions of the boundary layer of only some nanometers the process must lead to a sufficient amount of copolymers by interdiffusion and transreaction. Only those chemical reaction types will become technically relevant which satisfy these requirements.

Among the reaction types that were intensively studied in the past are the coupling reactions of monoendfunctionalized polymers A and B forming simple diblock copolymers $\mathrm{AB}$ (Link). These reactions will lead only to relative low formation rates at the interface between the polymers because of the low concentration of the reactive groups. Furthermore, the variety of reaction products is restricted to diblock copolymers [11-13]. On the other hand, polycondensates may have many functional groups within the polymer chain and, therefore, give rise to more complicated reaction types like splitting (Split) or branching reactions (Branch), which also may be combined. Due to the higher concentration of functional groups compared to the simple Link reaction, these latter reaction types are expected to yield greater reaction rates and therefore become more important for building in situ reaction products in the twocomponent injection molding process [14]. Because of the small time and space dimensions being available for these reactions experimental investigations are very difficult to perform. For that reason theoretically analytical approaches and also simulation techniques seem to be useful methods to 
get some deeper insight into the fundamental statistical processes leading to bond formation in the interface region.

However, due to the simplicity of the reaction mechanism the reaction type Link, considering only low concentrations of reactive monomers, was subject to several analytical [15-18] and simulation [19, 20] studies. The other two types Split and Branch were investigated [21] by means of simulations only less and only more qualitatively so far. On the other hand, the above-mentioned polymer processing technologies require high growth rates of the reaction products and, therefore, must be performed at high concentrations of reactive chains.

Because of their simplicity maintaining the basic features of polymeric melts many simulations were performed by means of Monte-Carlo techniques using the Bond Fluctuation Model (BFM) [22-25]. This coarse-grained threedimensional lattice model retains the important features of a polymeric material like (i) connectivity of the monomers, (ii) excluded volume interaction, (iii) a short range thermal interaction potential, and allows to simulate essential properties of dense polymeric melts including the crossover behaviour from Rouse to reptation dynamics [32].

Using the BFM, we qualitatively investigated in a previous study [21] fast (activation energy $E_{A}=0 k_{B} T$ ) interface reactions between immiscible reactive polymers including complex reaction mechanisms like interchain and intrachain transreactions. Here, the kinetics of formation of reaction products (including ring copolymers) is no unique function of the consumption of reactive monomers $\mathrm{C}$ forming the ends of the B polymers. But this is necessary in the attempt to develop a quantitatively phenomenological kinetic model of (in the first stage) the as possibly simple, but practically relevant reaction types.

Therefore, we quantitatively investigated in a later work [26] the interface reaction behaviour $\left(E_{A}=0,1,3\right.$ and $\left.5 k_{B} T\right)$ between two immiscible polymers A and B exhibiting the reaction types Link, Split and Branch under technically relevant concentrations of reactive chains. It was shown, that the very first stage of the reaction in a good approximation could be described by a bimolecular time rule. Several products of the reaction Split are able to penetrate the volume B phase and are expected to extraordinarily contribute to the adhesion performance of the composite in the two-component injection molding.

Recently [27], we studied the reactive compatibilization (blending) of randomly functionalized backbone polymers with end-functionalized graft polymers forming cocontinuous nanostructured microphases. We have compared reacted and non-reacted systems under the same conditions. For the non-reacted system at low temperatures phase separation was observed. In the system with grafting reactions macroscopic phase separation was inhibited even if the consumption of reactive sites was only $50 \%$. The calculated structure factor indicated a distinct difference between the two simulation states in accordance with the $3 \mathrm{D}$ visualization of the system and the box-counting method.

Because of the high concentration of reactive monomers and the ability of different reaction products to penetrate the volume phase, interface reactions of the Split type were proved to be favouring candidates for compatibilization intentions [28]. We have also investigated modified reaction forms of this type.

However, in all the cases the relation between the chemical reaction types on microscopic level and the consequences concerning the macroscopic strength behaviour is not well understood. There are several concepts relating structure to strength resting on the assumption, that the greater the amount of mixing or chain interpenetration at the interface is, the greater will be the strength, developed by this structure [2]. Therefore, bridge concepts counting (i) the number of intersecting chains, (ii) the number of bridges crossing the interface, (iii) the average monomer interpenetration depth, or (iv) the number of monomers crossing the interface seem to be useful to develop functions describing macroscopically measurable properties on the molecular level. Therefore, and to complete a series of interface reaction studies, in our present investigation we compare the relative strength of composites created without reaction, and as result of two different interface reaction types - the network-building capable reaction Split-All and the catalytic interface reaction Split$K a t-$ at $E_{A}=0,1,3$ and $5 k_{B} T$ between functionalized immiscible polymer melts, respectively.

Experimental investigations [10] have shown, that the effect of interface reactions during the two-component injection molding process is high, when the reacting polymer molecules contain more reactive sites, like in the system Polycarbonate/Polyethyleneimine. On the other hand, the consequences for the compound adhesion are not quit clear up to now. Therefore, the model reaction Split-All exhibiting several reactive sites on both reactive polymers is used to simulate this particular reaction behaviour.

Finally, catalytic reactions are known to accelerate chemical consumptions, because they can decrease the activation energy performing a modified reaction mechanism than in the original process. So, transesterification reactions often are promoted by the addition of low molecular weight titanium compounds [29], which are applied with only low concentrations to avoid negative effects in the resulting polymeric products. But due to the low mobility of monomers in the polymeric melt and because the activation step also is a (in general) second order reaction, the consequences for the resulting total reaction consumption and the effects in compound adhesion are unknown. The reaction model Split-Kat was designed to get a first impression of the reaction behaviour within such a complex mechanism.

For the purpose of strength description we have adopted several bridge functions describing the degree of interpenetration on the molecular level and we calculate these properties from the results of Monte-Carlo simulations as function of time using the Bond Fluctuation Model.

Even though in practical polymer chemistry the number of reactive sites per chain is restricted, we have consciously decided to make all the monomers of one chain type (A and/or B) reactive to calculate the possible effects of strength enhancement most clearly pronounced. The aim of our work is to estimate the value of the chemical reaction types to cre- 
ate composites of adequate strength during the twocomponent injection molding process.

\section{MATERIALS AND METHODOLOGY}

Within the framework of the BFM [22-25] each segment allocates the eight corners of a unit cell in a simple cubic lattice with lattice unit $a_{0}$ and no site can be doubly occupied. All the length data, given in the text, will be in units of $a_{0}$. Bond vectors connect the centres of the cubes. Following the terminology of Carmesin and Kremer [22] we call the repeating units of the coarse-grained Monte Carlo model monomers. Serving the condition of the excluded volume and preventing the bond vectors from crossing during the random motion of the monomers leads to a set of 108 bond vectors with length squares of 4, 5, 6, 9 and 10 including 87 bond angles altogether.

Energetically we distinguish between only two monomer types, which we call $\mathrm{A}$ and $\mathrm{B}$, respectively. The short-range pair interaction potential will be calculated usually between the monomer units and the surrounding 54 segments in a distance of $r \leq \sqrt{6}$ with an energy term $\varepsilon=\varepsilon_{A B}=-\varepsilon_{A A}=-\varepsilon_{B B}$. In this way, monomers of the same type attract and monomers of different type reject each other.

The attempted move of a monomer unit will only be accepted, if (i) the excluded volume is retained, (ii) the new bonds are allowed in the BFM and (iii) the change in energy satisfies the Metropolis criterion [30]. We have decided to create a model of the immiscible A/B system consisting of 876 chains with degree of polymerisation of $P=32$ for each of the monomer types $\mathrm{A}$ and $\mathrm{B}$, respectively. The chains are contained in a box of size $L_{X} \cdot L_{Y} \cdot L_{Z}=(-120:+120)$. $(-30:+30) \cdot(-30:+30)$ with 56064 monomers representing a volume fraction of nearly 0.5 , for which the BFM reproduces many characteristic properties of a dense polymeric melt.

Periodic boundary conditions were imposed on all three spatial directions to simulate a system of infinite size. In this way, a sufficiently large diffusion path in $x$ direction is provided to ensure the unperturbed volume conditions for comparison intentions. Additionally, the periodic boundary conditions in $x$ direction create $1+2 \cdot \frac{1}{2}=2$ interfaces, which act as a doubled reaction surface. The initial configuration was built from 56064 identical monomers and equilibrated without the energy criterion using $10^{6}$ Monte-Carlo steps (MCS). Then, the chains were separated into phases of type A and B depending on the $x$-coordinate $x_{\text {com }}$ of the centre of mass of the chain $\left(x_{\text {com }}<0: \mathrm{A}, x_{\text {com }}>0: \mathrm{B}\right)$, so that the mathematical interface between the A/B phases is the $y z$-plane at $x=0$.

The degree of polymerisation of $P=32$ is well below the value, where the first hints for reptation like motion are observed. We have set the energy parameter to be $\varepsilon=0.1 k_{B} T$ and so the incompatibility corresponds to $\chi P \approx 17$, which is well inside the strong segregation limit of a binary polymeric blend.
The configuration again was equilibrated using $10^{6} \mathrm{MCS}$ including the energy calculation within the Metropolis criterion to form out the equilibrium interface structure between the polymers until the static properties bond length $l$, end-toend distance $R_{E}$, and radius of gyration $R_{G}$ became constant.

From the final configuration, starting a simulation of appropriate duration, configurations were extracted nearly all $10^{4}$ MCS to be used as start configurations for the simulations including the chemical reaction. After a pre-reaction simulation run of about $10^{6}$ MCS the chemical reactions were initiated at $t=0$ (similarly, as we would use a radiation flash in the experiment to activate the reaction centres) and were processed more than $2 \cdot 10^{5}$ MCS (201813) just exceeding the Rouse time $\tau_{R}$ of chains with degree of polymerisation $P=32$. The time $\tau_{R}$ was estimated from a simulation run without reaction to be $\tau_{R} \approx 10^{5} \mathrm{MCS}$. The chemical reactions being under consideration in this study are non-reversible and are governed by activation energies of $E_{A}=0,1,3$ and $5 k_{B} T$, which are implemented in terms of an additional Metropolis criterion. To prepare the equilibrated system for the reaction intentions, some modifications are necessary and distinct monomers having now special tasks are marked by new names, but remain energetically of type A or B. For the non-catalytic reaction of Split type, which we now call Split-All, all monomers in the chains A and $\mathrm{B}$ are made reactive and are indicated as D and C, respectively. In this way, the products are able to continue the reaction forming new products, and so also highly branched molecules and even a network system (in the sense of highly branched molecules) should possibly be created. In Fig. (1a) the reaction mechanism is shown schematically.

For the catalytic reaction system, which now is called Split-Kat, all monomers in the A chains are denoted as $\mathrm{G}$ and are made reactive. In the $\mathrm{B}$ chains, the middle monomers are reactive only and are called $\mathrm{C}$. To create the free catalyst monomers within the same total monomer number density, from the $\mathrm{B}$ chains one end monomer was split up, and the whole system then was re-equilibrated. By means of special boundary conditions the free catalyst monomers $\mathrm{K}$ were restricted to move only within the B phase. So, the starting condition for the catalytic reaction consists of $\mathrm{G}$ chains with $P=32$ in the A phase, and $\mathrm{B}$ chains with $P=31$ including reactive $\mathrm{C}$ monomers mixed by free $\mathrm{K}$ monomers in the $\mathrm{B}$ phase. When the reaction is initiated, first in step (I) an arbitrary $\mathrm{G}$ monomer is converted into a $\mathrm{D}$ monomer by a $\mathrm{K}$ monomer and in this way is activated, whereas the catalyst monomer $\mathrm{K}$ is transformed into a free $\mathrm{L}$ monomer. The chains containing an activated monomer D now in step (II) can be randomly split by the attack of a reactive monomer $\mathrm{C}$ in the chains B usually forming an unsymmetrical diblock copolymer and a fragment of the $G$ chain. As subsequent reaction products also triblock copolymers or additional single monomers may arise. The just reacted monomers $\mathrm{C}$ and $\mathrm{D}$ are now denoted as (non-reactive) $\mathrm{E}$ and (still reactive) $\mathrm{F}$, respectively. Note, that in this way the consumption of reactive monomers $\mathrm{C}$ is a unique measure for the progress of the 
reaction and every consumption event of a $\mathrm{C}$ monomer leads to the formation of exactly one reaction product (or pair of products). Each time a reaction event occurred, in a log file the exact MCS was recorded to construct the non-localized consumption of reactive monomers $\mathrm{C}$ as function of time. All chains of type B do not split and in step (III) the remaining $\mathrm{F}$ monomer connecting the $\mathrm{G}$ fragment to the $\mathrm{B}$ chain is converted into a non-reactive $\mathrm{H}$ monomer by a free $\mathrm{L}$ monomer, which itself is transformed into a free $\mathrm{K}$ monomer closing the catalytic reaction circle. We have decided to fix the activation energy for the steps (I) and (III) at $E_{A}=0$ $k_{B} T$, but to vary the activation energy for step (II) according to $E_{A}=0,1,3$ and $5 k_{B} T$. The initial concentration level of free $\mathrm{K}$ monomers was set to $c_{K}^{0}=c_{C}^{0} \quad($ Split-Kat-A), $c_{K}^{0}=c_{C}^{0} / 4 \quad\left(\right.$ Split-Kat-B) and $c_{K}^{0}=c_{C}^{0} / 8$ (Split-Kat-C). In order to keep the total number density of monomers con- stant, we have deactivated the redundant $\mathrm{K}$ monomers in the systems Split-Kat-B and Split-Kat-C. In Fig. (1b) the catalytic reaction system is presented schematically.

The reaction in step (II) directly corresponds to the reaction type Split-Mid in our previous work [28], and so the observations for the new reaction scheme may be compared to the former obtained results.

Starting from statistically independent configurations, 100 simulation runs for each of the tasks were processed to ensure sufficiently statistical certainty. The simulations were performed on eight standard personal computers (Pentium IV, $2.8 \mathrm{GHz}, 512 \mathrm{MB}$ RAM) consuming 6 hours CPU time per simulation run ( $\approx 1.5 \cdot 10^{6}$ attempted moves per second). The $170 \mathrm{MB}$ data for the configurations at several MCS were stored on disk for further evaluation. The simulation program itself was written in DELPHI, for the extraction of sev-

a

\section{Split-All}

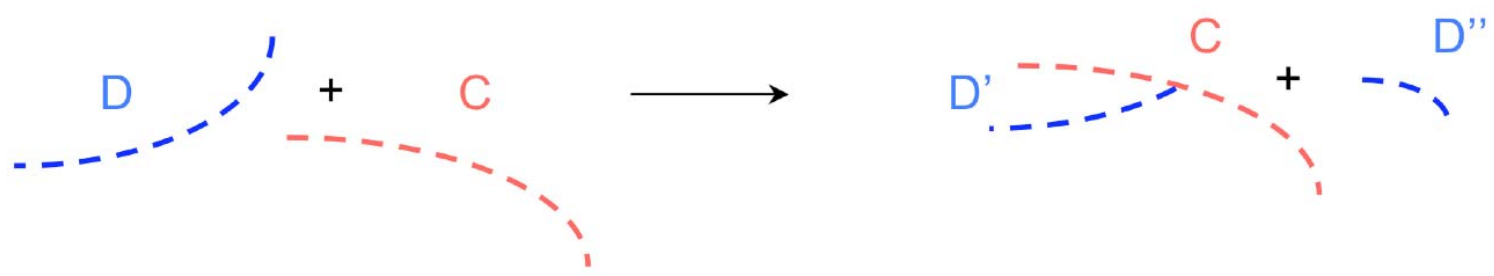

\section{- reactive monomers}

b

Step 1

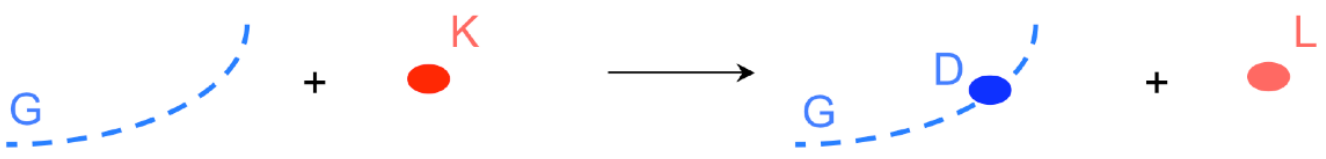

\section{Step 2}

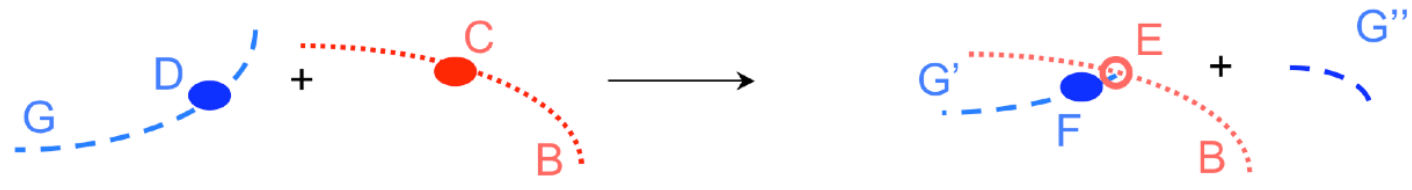

\section{Step 3}

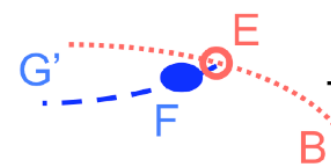

$\mathrm{B}$<smiles>C[C@H]1CC[C@H]1C</smiles>
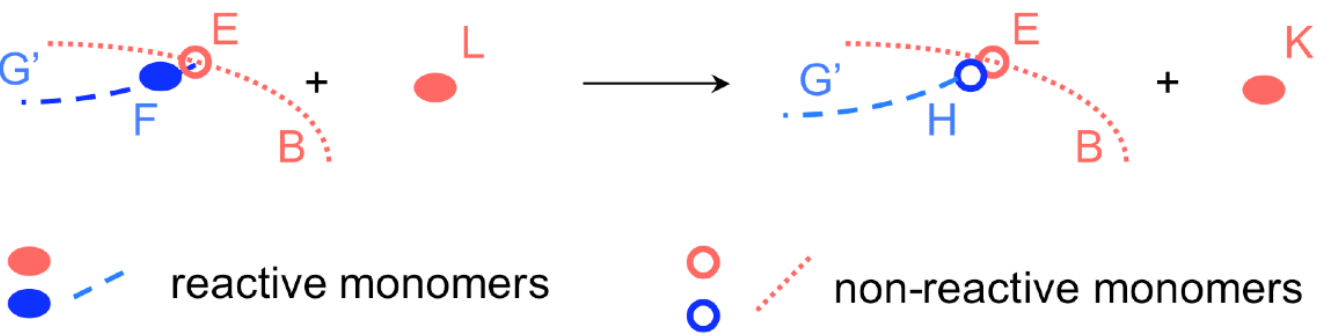

reactive monomers

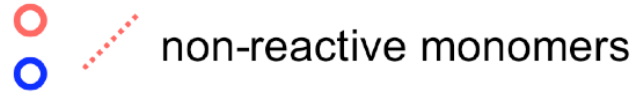

Fig. (1). Schematic representation of the reaction types Split-All (a) and Split-Kat (b). 
eral physical properties of the system like end-to-end distance, radius of gyration, displacements of single and multiple bound monomers and the reaction events as a function of time a Compaq Fortran90 program was written and used to process the configurations of one task at once.

\section{RESULTS AND DISCUSSION}

First, after equilibration of the two-phase system, we investigated three selected static properties of the demixed blend in an independent simulation run without reactions. For the reaction Split-All, the end-to-end distance $\left\langle R_{E}^{2}\right\rangle^{1 / 2}=16.9 \pm 0.8$, the radius of gyration $\left\langle R_{G}^{2}\right\rangle^{1 / 2}=6.9 \pm 0.3$ and the bond length $\left\langle\mathbf{l}^{2}\right\rangle^{1 / 2}=2.62 \pm 0.01$ are close to values from our previous work [26] $\left(\left\langle R_{E}^{2}\right\rangle^{1 / 2}=16.4,\left\langle R_{G}^{2}\right\rangle^{1 / 2}=6.8\right.$, $\left\langle\mathbf{l}^{2}\right\rangle^{1 / 2}=2.62$ and are somewhat less than those found for a homogeneous system of the same degree of polymerisation and volume fractions of the monomers without interaction of 17.1, 7.0 and 2.64 [31], respectively. Looking at the statistical uncertainties we believe these differences to be randomly. For the system Split-Kat due to the lower degree of polymerization of $P=31$ in the phase $\mathrm{B}$, we find somewhat lower values of the end-to-end distance $\left\langle R_{E}^{2}\right\rangle^{1 / 2}=13.7$ and the radius of gyration $\left\langle R_{G}^{2}\right\rangle^{1 / 2}=5.6$, but a little greater value for the bond length $\left\langle\mathbf{I}^{2}\right\rangle^{1 / 2}=2.63$. However, the physical properties follow the relation $\left\langle R_{E}^{2}\right\rangle \approx 6 \cdot\left\langle R_{G}^{2}\right\rangle$ and the number density of monomers is the same as in the case of the reaction Split-All, and so it is also the same as in previous work [26].

\section{CONSUMPTION}

In Fig. (2) we present a survey of all the consumption curves for the reactive $\mathrm{C}$ monomers as a function of time in a double logarithmic scale.

The lower the concentration of the catalyst in the system Split-Kat was applied, the lower was the resulting reaction rate in the sequence Split-Kat-A, Split-Kat-B and Split-Kat$C$. The greater the activation energy for the reaction Split-All and for step (II) of the catalytic reaction Split-Kat was adjusted, the more the onset of the reaction (with consumption $=1$ ) is shifted to larger times. Similar to formerly discussed results [26] we also find a typical appearance of the curves forming a first part at lower times with a slope of $\approx 0.8-1.0$ and a second part at later times with a slope of $\approx 0.2-0.3$ for all the reaction types. The first part of the curves (that we call kinetic controlled) we attribute to the consumption of reactive $\mathrm{C}$ monomers by local and frequent monomer contacts due to conformation alterations around the reaction zone at $x \approx \pm R_{G}$. Neglecting transport phenomena, this behaviour may be described by a simple bimolecular time rule $\frac{d n_{C}}{d t}=-k \cdot n_{C} \cdot n_{D}$ where $n_{C}$ and $n_{D}$ are the total numbers of

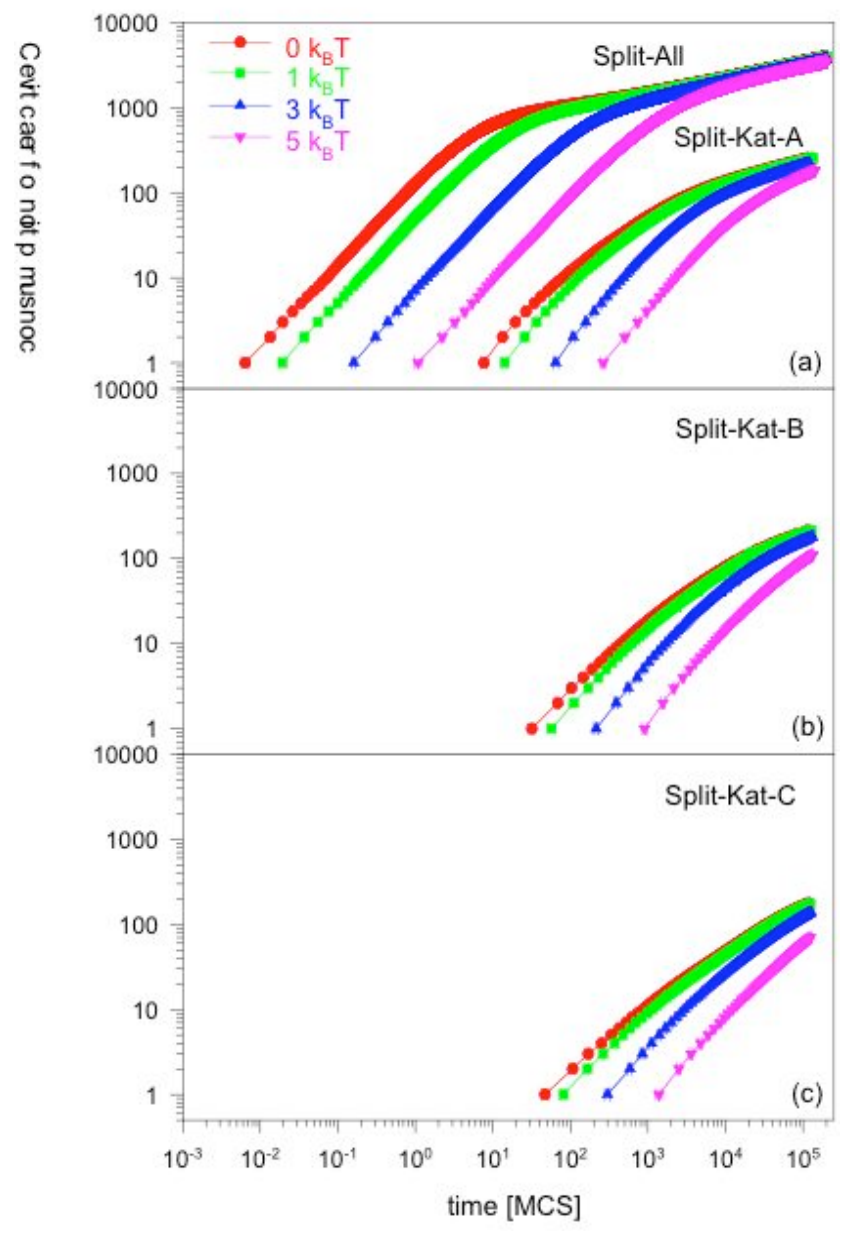

Fig. (2). Consumption of reactive $\mathrm{C}$ monomers for the reactions Split-All/Split-Kat-A (a), Split-Kat-B (b) and Split-Kat-C (c) at activation energies of $0,1,3$ and $5 k_{B} T$, respectively.

reactive monomers $\mathrm{C}$ and $\mathrm{D}$ being available in the reaction zone, respectively, and $k$ is the reaction rate constant. Clearly, this simple ansatz will not describe the crossover behaviour to the second part in time, but it provides the possibility to calculate the intrinsic kinetic rate constant of the process in the very first stage of the reaction. Because of the complexity of the catalytic reaction, the rate constant cannot be calculated directly from the bimolecular ansatz as described earlier [26]. But it can be estimated as an adjustable parameter being the part of a more sophisticated reactiondiffusion model, as described below. The second part (that we call diffusion controlled) we believe to be the result of transport processes of reactive monomers from the volume phases into the reaction zone by Ficks diffusion. In our opinion the slope of $\approx 0.2-0.3$ can not be explained in a simple straightforward manner based on the known displacement behaviour of the monomers. But it can be derived from the previously introduced reaction-diffusion model [26] $\frac{1}{a_{0}} \frac{\partial c_{C}}{\partial t}=D(t) \frac{\partial^{2} c_{C}}{\partial x^{2}}-k c_{C} c_{D}$, when the calculated monomer concentration profiles $c_{C}(x, t)$ for proper values of $D$ and $k$ are integrated. To obtain the predicted consumption curves for reactive $\mathrm{C}$ monomers for each configuration (time step) 
we calculate the total number $n_{C}(t)=\int_{-120}^{+120} c_{C}(x, t) d x$ of nonreacted $\mathrm{C}$ monomers and create the difference to the value at the origin in time $u_{C}(t)=n_{C}^{0}-n_{C}(t)$ - the consumption. As we found numerically, the curves $u_{C}(t)$ exhibit for small times in the doubly logarithmic scale a slope of $<1$, but in later times below the Rouse time a slope of $\approx 0.2-0.3$, as to be observed in the simulations.

\section{DEGREE OF POLYMERIZATION}

We present in Fig. (3) the distribution of the degree of polymerization (the molar fraction $\varphi(P)$ for molecules of degree of polymerization $P$ as function of $P$ ) after termination of the simulation at the time $t=201813$ MCS narrow above the Rouse time.

For the two reaction types Split-All and Split-Kat-A, the distributions of the degree of polymerization in the range $1 \leq P \leq 32$ is similar and we find maxima at $P=32$ for both the reactions and $P=66$ for Split-Kat-A only. Free monomers $P=1$ in the system Split-Kat-A are present from the beginning as catalyst monomers $\mathrm{K}$ and in the system SplitAll they may arise from the attack of a $\mathrm{B}$ chain with reactive monomers $\mathrm{C}$ at the second last monomer of an A chain. The molecules in the range $1<P<32$ are fragments of divided A chains, since the $\mathrm{B}$ molecules remain indestructibly. The maximum at $P=32$ is due to non-reacted $\mathrm{A}$ and $\mathrm{B}$ chains, whereas the products $33 \leq P \leq 64$ are $\mathrm{B}$ chains connected to fragments of A up to symmetric diblock copolymers at $P=$ 64. The latter are formed relatively frequent due to the enlarged mobility of the end monomers of the chains. Finally, the molecules at $65 \leq P \leq 96$ are triblock copolymers consisting of two B chains and variably long A fragments up to symmetric triblock copolymers with $P=96$. This is also the upper limit of the degree of polymerization for the reaction type Split-Kat. The reason for the relative maximum at $P=$ 66 in the system Split-Kat-A is the result of the fact, that a triblock copolymer containing two innermost A monomers cannot be split anymore and so it is enriched as a final product at our termination time $t=201813 \mathrm{MCS}$. In contrast to the reaction Split-Kat-A, for the reaction Split-All the molar fraction of molecules with $P>32$ strongly decreases with growing degree of polymerization for the benefit of the creation of very large polymers in the range $5000 \leq P \leq 7000$ leaving a broad gap between $P \approx 100 \ldots 5000$. These molecules can be formed, since all monomers in the type $\mathrm{A}$ and $\mathrm{B}$ chains are reactive and also remain reactive as part of reaction products, as long as they are not have been consumed by the progressing reaction. In this way large structures like felts, which also can contain cyclic constructs, can arise along the interface, and an example of such a giant molecule is shown in Fig. (4) together with a snapshot of the simulation box.

Since the polymer arms of energetically type A and B can be embedded within the corresponding chemical phases, a synergistic effect for the enhancement of tensile strength may be expected.

\section{CONSUMPTION PER MONOMER}

The consumption curves in Fig. (2) were built from a log file, which was created during the simulation run recording the time (MCS) of each reaction event indicating the consumption of a $\mathrm{C}$ monomer. On the other hand, from the concentration profiles $c(x, t)$ for each monomer within the simulation box, which were constructed from the evaluation of the stored configurations, the consumption curves for each of the monomers can be calculated by integration over the $x$ coordinate. In Fig. (5) we present these consumption curves, which are now only a function of time and which give some overview about the progress of the catalytic reaction system.

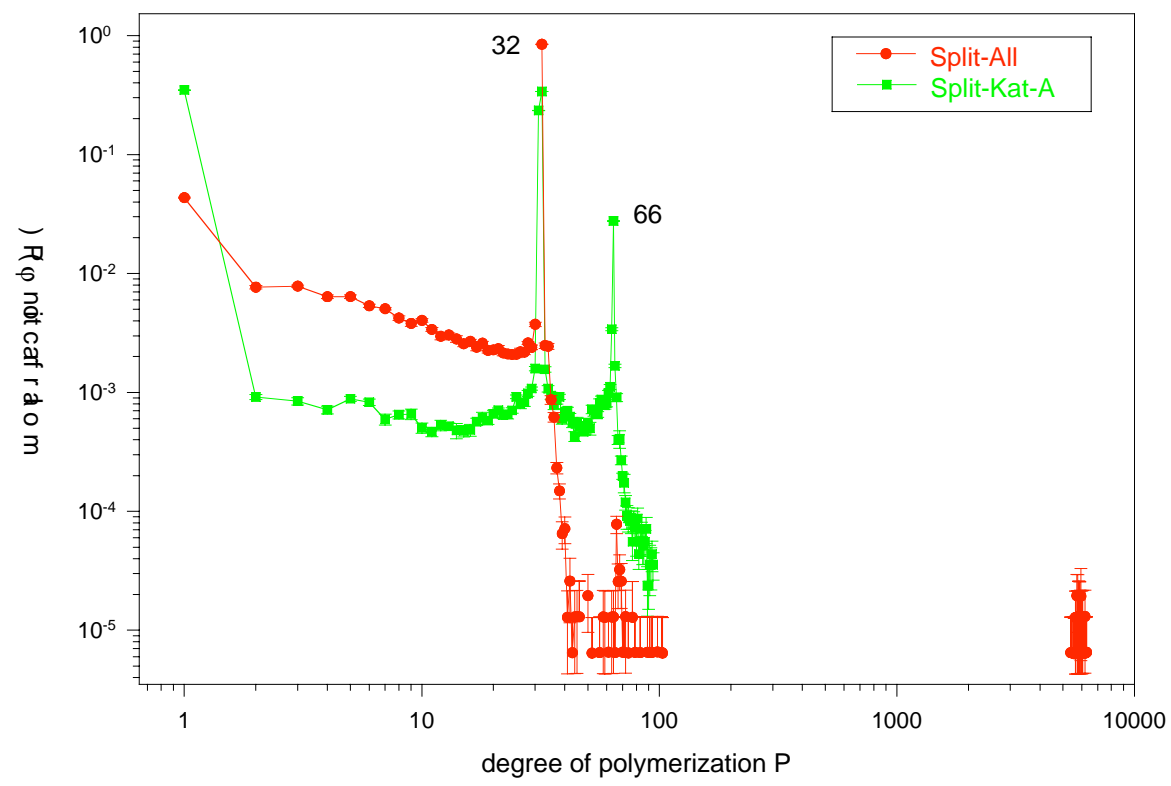

Fig. (3). Distribution of the degree of polymerization for the reactions Split-All and Split-Kat at activation energy $E_{A}=0 \quad k_{B} T$ after termination of the simulation at time $t=201813$ MCS. 

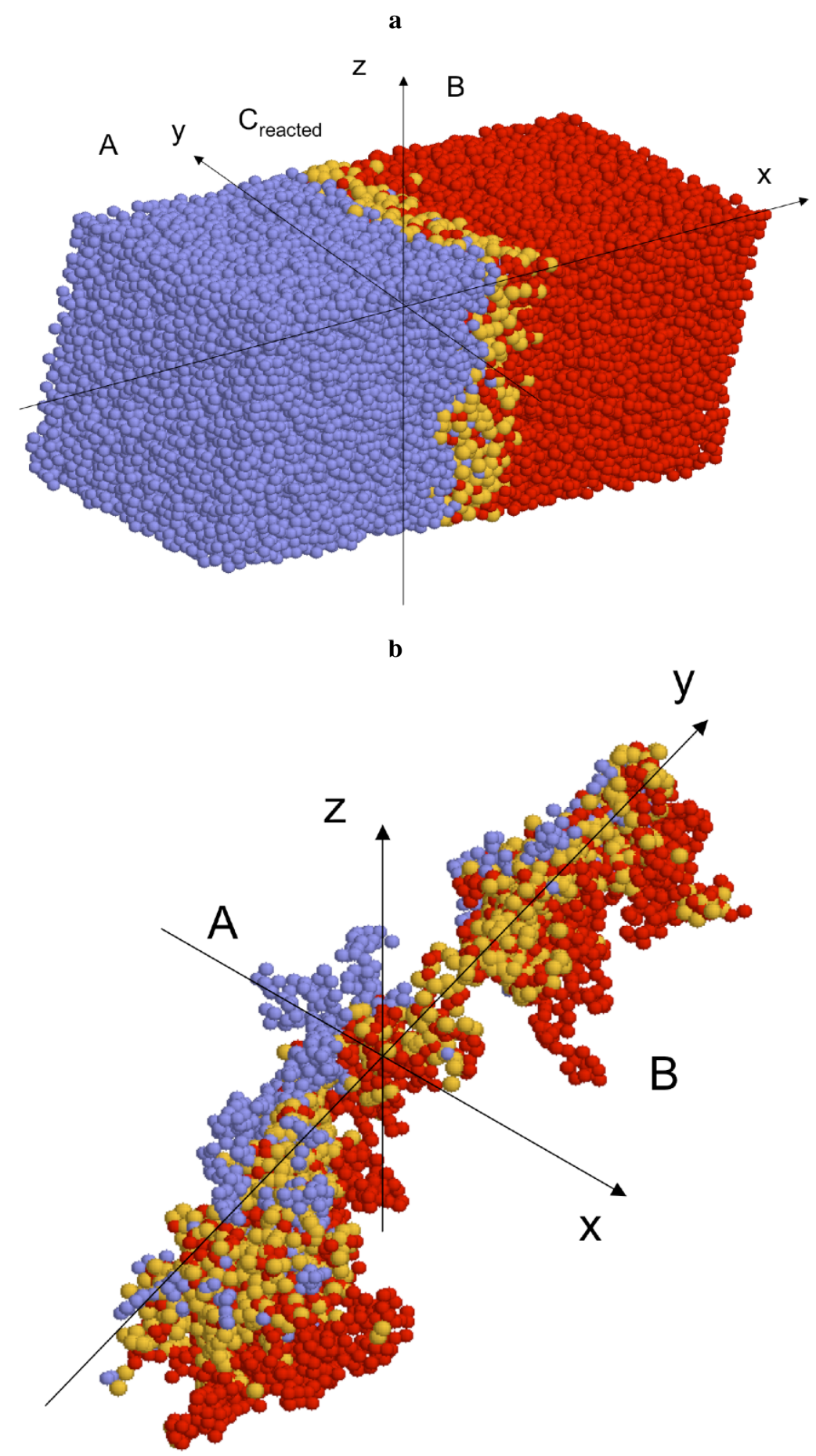

Fig. (4). Snapshot of the simulation box (a) and a product molecule with degree of polymerization of $P=5054$ (b) of the reaction $S p l i t-A l l$ at time $t=30034$ MCS.

According to the reaction scheme in Fig. (1b), G monomers near the interface region in step (I) are activated by free $\mathrm{K}$ monomers (the catalyst) to form active $\mathrm{D}$ monomers as reaction partners for the main interface reaction step (II). Since the $G$ monomers are present in great excess and the free $\mathrm{K}$ monomers are very mobile, this process is fast and leads to an increase of the number of active $\mathrm{D}$ and the free $\mathrm{L}$ monomers (the curves nearly coincide). The latter ones are needed later to regenerate $\mathrm{K}$ monomers within the catalytic cycle. Only about 25 chains of $\mathrm{G}$ would be consumed to transform all the initially distributed $\mathrm{K}$ monomers completely, so that the total number of G in Fig. (5) is only slightly decreased. At the interface the molecules containing $\mathrm{C}$ and D monomers now can react in step (II) to form stable $\mathrm{E}$ and the still reactive $\mathrm{F}$ monomers labelling the contact position of the (not divided) B chain and the A fragment. The $\mathrm{F}$ monomers in step (III) are transformed into nonreactive $\mathrm{H}$ monomers consuming $\mathrm{L}$ and regenerating the catalytic $\mathrm{K}$ monomers. This process is slightly shifted to a greater time (MCS 4) and later the curves for $\mathrm{E}$ and $\mathrm{H}$ 


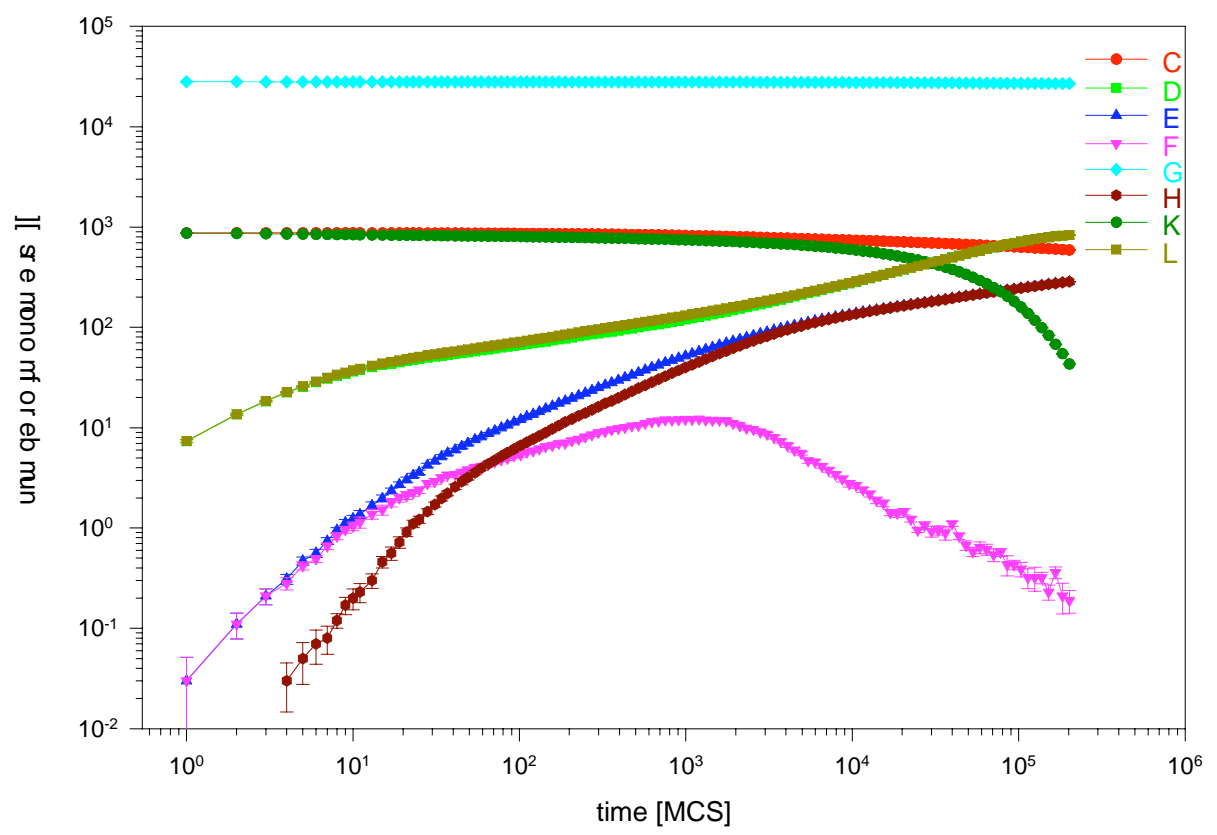

Fig. (5). Total number of monomers for the reaction Split-Kat-A at activation energy $E_{A}=0 \quad k_{B} T$ as function of time.

monomers become identically. Since the interface reaction is relatively slow, practically all $\mathrm{F}$ monomers are consumed and the remaining excess $\mathrm{L}$ monomers because of their high mobility are able to fill the whole box, as it is also visible in the concentration profiles discussed below. Due to the relatively slow formation of $\mathrm{F}$ monomers as result of the interface reaction (II), the regeneration rate of $\mathrm{K}$ monomers appears delayed and the total number of $\mathrm{K}$ monomers strongly decreases for greater simulation times as consequence of the volume reaction (I). To illustrate the overall consumption behaviour, we have calculated the total variation $\Delta n$ of the number for each of the monomers within the time being discussed in Fig. (5): dC $=-285((-)$ total number of consumed $\mathrm{C}$ monomers $), \mathrm{dD}=+834((+)$ number of activated $\mathrm{G}$ minus number of consumed $\mathrm{D}$ ( = number of consumed $\mathrm{C})$ ), $\mathrm{dE}=$ +285 ((+) number of stable $\mathrm{E}$ formed by 285 consumed $\mathrm{C})$, $\mathrm{dF}=0$ (as formed, so consumed), $\mathrm{dG}=-1119((-)$ total number of activated $\mathrm{G}), \mathrm{dH}=+285((+)$ number of stable $\mathrm{H}$ formed by 285 consumed $\mathrm{C}), \mathrm{dK}=-834((-)$ number of activated $\mathrm{G}$ minus number of rebuilt $\mathrm{K}=$ number of converted $\mathrm{H}), \mathrm{dL}=+834((+)$ number of activated $\mathrm{G}$ minus number of formed $\mathrm{H})$. Later we will discuss the rate relation between steps (I) - (III) by means of a simple reaction-diffusion model qualitatively.

\section{CONCENTRATION PROFILES}

In Fig. (6) we have compiled the concentration profiles $c(x)$ for the monomers in the system Split-Kat-A after termination of the simulation at time $t=201813 \mathrm{MCS}$.

According to the reaction mechanism in Fig. (1b), the G monomers near the interface, which are in contact to catalytic $\mathrm{K}$ monomers, are activated and transformed into $\mathrm{D}$ monomers. Because the $\mathrm{G}$ monomers are present in a great excess, there concentration profile is only less modified compared to $t=0 \mathrm{MCS}$. The $\mathrm{K}$ monomers are consumed near the interface, but due to their great mobility also the concentration in the volume B phase decreases and the D monomers become enriched near the interface. The regeneration of the $\mathrm{K}$ monomers by $\mathrm{L}$ monomers within the catalytic cycle seems to be disturbed, as it will be discussed below. The activated $\mathrm{D}$ monomers are located in the interface region and can react with $\mathrm{C}$ monomers within this spatial area to form a copolymer containing now the contact monomers $\mathrm{E}$ (from $\mathrm{C}$ ) and $\mathrm{F}$ (from $\mathrm{D}$ ), whereas a fragment of the $\mathrm{G}$ chain is released. As result of this process the concentration profile of the non-reacted $\mathrm{C}$ monomers is shifted into the volume $\mathrm{B}$ phase. The reaction products di- or triblock copolymers remain within the interface region and their position is labelled by the position of $\mathrm{E}$ (being now non-reactive) and $\mathrm{F}$ monomers. These $\mathrm{F}$ monomers are transformed to non-reactive $\mathrm{H}$ monomers by $\mathrm{L}$ monomers regenerating the $\mathrm{K}$ monomers in the catalytic cycle. The concentration of the intermediate $\mathrm{F}$ monomers is only very low, because they are instantaneously transformed into $\mathrm{H}$ monomers by $\mathrm{L}$ monomers being nearly homogeneously distributed within the volume phases. The L monomers are formed in step (I) at the interface by reaction of a large excess of $G$ monomers with the catalyst $K$ (enrichment of D). Because of their great mobility the L monomers can penetrate the volume phases until they are consumed by $\mathrm{F}$ monomers as component of just generated block copolymers in step (II). Since this reaction may only be performed at the interface, only a small part of the L monomers are converted and so the regeneration rate of the catalyst $\mathrm{K}$ will be delayed. The distribution of non-reactive $\mathrm{E}$ monomers (energetically of type B) is shifted somewhat into the direction of the $B$ phase, and the profile of non-reactive $H$ monomers corresponding to their energetically type $\mathrm{A}$ is located more within the A phase. Particularly considered, in contrast to the $\mathrm{E}$ concentration the profile of the $\mathrm{H}$ monomers exhibits a double peak which is caused by A chain fragments of different size in diblock- and triblock copoly- 


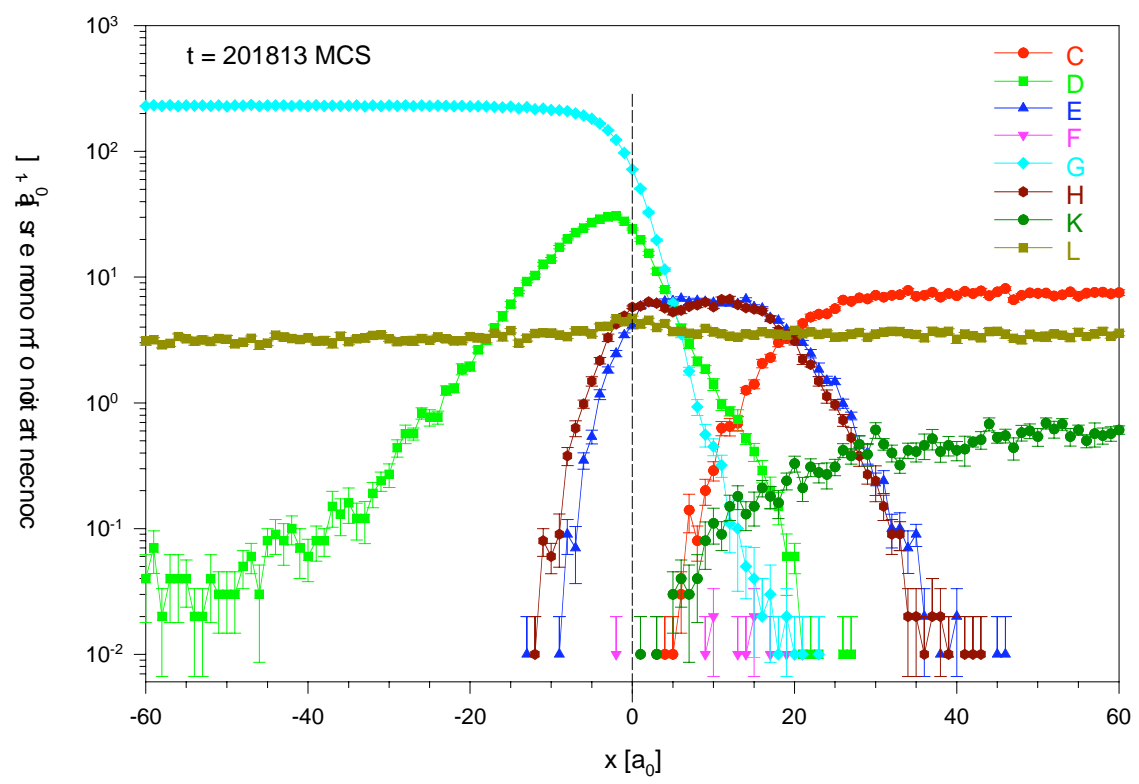

Fig. (6). Concentration profiles of the monomers for the reaction Split-Kat-A at activation energy $E_{A}=0 \quad k_{B} T$ after termination of the simulation at time $t=201813$ MCS.

mers, as it was also found earlier for simple interface reactions [28].

\section{REACTION-DIFFUSION MODEL}

To predict the concentration profiles for the monomers in the reaction Split-Kat qualitatively, we have extended our previously developed reaction-diffusion model [26]. For each of the eight monomers $M$ the partial differential equation for the alteration of the concentration in time $t$ and direction $x$ is constructed from Ficks second law expanded by a reaction term as source or depression according to the scheme in Fig. (1b) as

$\frac{\partial c_{M}}{\partial t}=\frac{\partial}{\partial x}\left[D_{M}(t, x) \cdot \frac{\partial c_{M}}{\partial x}\right]+R_{M}$

with the reaction expressions

$R_{C}=-k_{2} \cdot c_{C} \cdot c_{D}$

$R_{D}=+k_{1} \cdot c_{G} \cdot c_{K}-k_{2} \cdot c_{C} \cdot c_{D}$

$R_{E}=+k_{2} \cdot c_{C} \cdot c_{D}$

$R_{F}=+k_{2} \cdot c_{C} \cdot c_{D}-k_{3} \cdot c_{F} \cdot c_{L}$

$R_{G}=-k_{1} \cdot c_{G} \cdot c_{K}$

(2),

$R_{H}=+k_{3} \cdot c_{F} \cdot c_{L}$

$R_{K}=-k_{1} \cdot c_{G} \cdot c_{K}-k_{3} \cdot c_{F} \cdot c_{L}$

$R_{L}=+k_{1} \cdot c_{G} \cdot c_{K}-k_{3} \cdot c_{F} \cdot c_{L}$

which leads to the formation of a system of coupled partial differential equations. We have decided to separate the diffusion functions according to the valence state of the relevant monomers into non-bound (superscript 0 ) and bound (superscript 2) environment:

$D_{C}(t, x)=D_{D}(t, x)=D_{E}(t, x)=D_{F}(t, x)=D_{G}(t, x)=$

$D_{H}(t, x)=D_{x}^{2}(t, x)$

$D_{K}(t, x)=D_{L}(t, x)=D_{x}^{0}(t, x)$
The non-zero initial and marginal conditions are

$c_{C}(0, x)=c_{C}^{0} \cdot\left[1-\frac{1}{1+\left(\frac{x+121}{121}\right)^{60}}\right]$

$c_{G}(0, x)=\frac{c_{G}^{0}}{1+\left(\frac{x+121}{121}\right)^{80}}$

$c_{K}(0, x)=c_{K}^{0} \cdot\left[1-\frac{1}{1+\left(\frac{x+121}{121}\right)^{60}}\right]$

(fitting the simulated profiles of the $\mathrm{C}, \mathrm{G}$ and $\mathrm{K}$ monomers) and

$c_{C}(t, 120)=c_{C}^{0}$

$c_{G}(t, 0)=c_{G}^{0}$

$c_{K}(t, 120)=c_{K}^{0}$

with

$c_{C}^{0}=7.3 a_{0}^{-1}$

$c_{G}^{0}=233.6 a_{0}^{-1}$

$c_{K}^{0}=7.3 / 1.825 / 0.9125 a_{0}^{-1} \quad(A / B / C)$

(for three concentration levels of the catalyst K). The reaction rate constants are expressed as Arrhenius relation $k_{i}=k_{0} \cdot e^{-\frac{E_{A i}}{k_{B} T}}$. We first specify the activation energies $E_{A i}=0 k_{B} T$. The pre-exponential factor $k_{0}$ is processed as 
(the only) adjustable parameter to fit the monomer $\mathrm{C}$ profile in the reaction Split-Kat-A for $E_{A}=0 k_{B} T$ after terminating the simulation at $t=201813$ MCS (see below). Following the concept of our previous work [26], we have decided to extract the diffusion properties for the numerical solution of the system of partial differential equations (1) from the simulation data and to fit them to properly defined analytical functions. Consequently, we only consider the motion in $x$ direction and the monomers are distinguished in non-bound (valence $=0$ ) and bound (valence $>0$ ) monomers. From the simulation run for the system Split-Kat-A without reaction the diffusion functions were calculated from the mean square displacements $\left\langle(x(t)-x(0))^{2}\right\rangle=f(t, x)$ for both the monomer types using the Einstein-Smoluchowski relation [33] $\left\langle\Delta x^{2}\right\rangle=2 \cdot D_{x}(t, x) \cdot t$. In the following, the parameters and functions for non-bound monomers are indicated by a superscript zero (0) and those for bound monomers by a superscript two (2). For the non-bound monomers, first the diffusion function $D_{x}^{0}(t)$ was calculated only as a function of time as average across the simulation box. The predominant behaviour of this function within the simulation time can be attributed to the propagation of free $\mathrm{K}$ monomers around the whole simulation box, which at $t=0$ were restricted to the B phase only. For convenience, we have decided to divide the diffusion data of the whole simulation time range into two sections, which then were fitted to polynoms of degree three (for $t<520 \mathrm{MCS}$ ) and fife (for $t \geq 520 \mathrm{MCS})$ in $\log (t)$, respectively. So, the only time dependent diffusion function for the non-bound monomers reads

$$
D_{x}^{0}(t)=\left[\begin{array}{ll}
t=0 & D_{x}^{0}=0.03 \\
0<t \leq 520 & \log \left(D_{x}^{0}\right)=P_{3}^{0}(\log (t)) \\
t>520 & \log \left(D_{x}^{0}\right)=P_{5}^{0}(\log (t))
\end{array}\right]
$$

the values of the polynom coefficients are given in the appendix. When we now additionally resolve the diffusion data as a function of the position $x$ of the monomers in the simulation box, the time and spatial dependent function $D_{x}^{0}(t, x)$ can be derived as a fit function of the form

$$
D_{x}^{0}(t, x)=D_{x}^{0}(t)+A^{0} \cdot \sin \left(\frac{x+120}{240} \cdot 2 \pi\right)
$$

were the amplitude $A^{0}$ was adjusted by a quadratic polynom

$$
A^{0}=\frac{1}{2} P_{2}^{0 A}(t)
$$

in the simulation time $t$ (MCS). Clearly, this function only can convey a rough impression of the diffusive behaviour of the non-bound monomers, but this surely should be adequate to the more qualitative picture, which is outlined by the reaction-diffusion model (1). In a similar manner we have calculated the time dependent diffusion function $D_{x}^{2}(t)$ for the bound monomers from the appropriate mean square displacements. As expected, the nearly exponential decay leads to a quasi-linear behaviour in the double logarithmic scale, and for fitting purposes we have also decided to separate the time range into two sections:

$$
D_{x}^{2}(t)=\left[\begin{array}{ll}
t=0 & D_{x}^{2}=0.03 \\
0<t \leq 520 & \log \left(D_{x}^{2}\right)=P_{3}^{2}(\log (t)) \\
t>520 & \log \left(D_{x}^{2}\right)=P_{2}^{2}(\log (t))
\end{array}\right]
$$

where we have fitted the logarithm of $D_{x}^{2}$ by appropriate polynoms of the logarithm of time. To get access to the spatial dependency of the diffusion function we have evaluated the corresponding mean square displacements of the twicebound monomers at three different steps in time. In this case we do not find a pronounced minimum of the diffusion function at $x \approx 0$ indicating the restricted mobility of the monomers perpendicular to the phase boundary, but two low depressions on both sides of the interface. Therefore, we have developed a model function for this diffusion $D_{x}^{2}(t, x)=\left[\begin{array}{ll}x \leq-E & D_{x}^{2}=D_{x}^{2}(t) \\ -E<x<+E & D_{x}^{2}=D_{x}^{2}(t)-A^{2} \cdot\left(1-\cos \left(\frac{x}{E} \cdot 2 \pi\right)\right) \\ x \geq+E & D_{x}^{2}=D_{x}^{2}(t)\end{array}\right]$

where the amplitude $A^{2}$ is a measure for the oscillation level and the elongation $E$ describes the extension of the oscillation in $x$ direction. Since we abstain from artificially creating a diffusion hole around the interface and we assume the evaluated data to be responsible, the model (1) will obviously overestimate the transport of monomers through the interface of the actually immiscible polymer phases. For simplicity, we have expressed the amplitude and elongation as linear functions of time:

$$
\begin{aligned}
& E=P_{1}^{2 E}(t) \\
& A^{2}=\frac{1}{2} P_{1}^{2 A}(t)
\end{aligned}
$$

Based on this input data, the system of coupled partial differential equations (1) can be solved numerically [34]. For this intention we have written a Fortran90 program using a Compaq development environment. Note, that in some cases depending on the choice of the parameter values, the numerical stability of the solution can be endangered. In these cases the time step width must be adjusted as low as necessary to avoid numerical fluctuations in the functions $c(t, x)$. For the more complex reaction Split-Kat the simple model of reaction zone of our previous work [26] is not applicable. Therefore, and in contrast to the procedure described earlier, we have estimated the reaction rate constant $k_{0}$ as (the only) adjustable parameter fitting the monomer $\mathrm{C}$ profile for the reaction Split-Kat-A at $E_{A}=0 k_{B} T$ after terminating the simulation at $t=201813 \mathrm{MCS}$.

For further discussions, we will know mainly focus on results, which we have obtained for the activation energy $E_{A}=0 k_{B} T$ and the reaction Split-Kat-A, since all the effects of the chemical reaction will be most pronounced in 


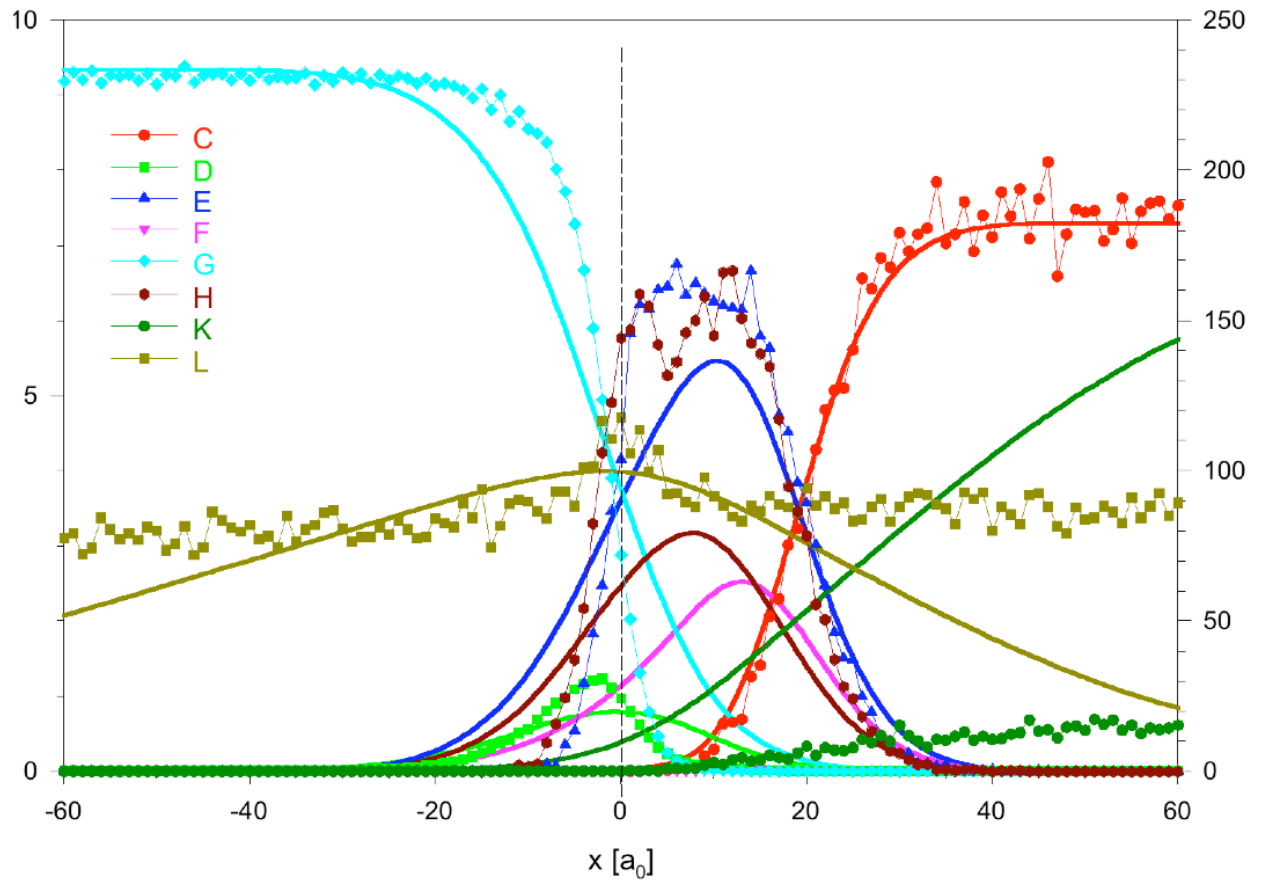

Fig. (7). Comparison of simulated concentration profiles (symbols and lines) of the monomers in the system Split-Kat-A with activation energy $E_{A}=0 \quad k_{B} T$ and calculated profiles (lines) of the reaction-diffusion model (1) at termination time of the simulation $t=201813 M C S$.

this case of the largest rate constant and catalyst concentration of the reaction type, being under consideration.

In Fig. (7) we have compiled a comparison between the simulated concentration profiles for the reaction Split-Kat-A for $E_{A 2}=0 k_{B} T$ at $t=201813 M C S$ and the calculated curves from the qualitative model (1) for $E_{A 1}=E_{A 2}=E_{A 3}=0 k_{B} T$ and the basic reaction rate constant $k_{0}=2.5 \cdot 10^{-6} a_{0} M_{C S}^{-1}$.

This reaction rate constant is in the order of magnitude of the rate constants, which we have calculated earlier [26] for some similar reaction types. As discussed above, we have estimated the model constant $k_{0}$ from the best approximation of the simulated concentration profile for $\mathrm{C}$ monomers by the calculated model curve. Therefore, the conformance with the simulated data for this curve is quite quantitatively. The calculated concentration profiles for the other monomers yield a more qualitative picture of essential features of the simulated profiles, particularly all calculated curves are broadened by an overestimation of the diffusive transport. The monomer D curve, like the simulated profile, is slightly shifted into the energetically (chemical) A phase, and the corresponding $\mathrm{E}$ profile (which arises from $\mathrm{C}$ monomers) is more located on the right hand (the B) side of the immiscible system. The greatest disagreement between simulated and calculated profiles we find for F monomers, which are hardly visible in the simulation, but are well pronounced right to the interface in the model calculation. We will discuss this effect by a variation of the model activation energies later in this section. The calculated $\mathrm{G}$ profile is broadened by diffusion and due to the boundary conditions well fits the simulated data within the volume phase. The calculated curve for the resultant and non-reactive $\mathrm{H}$ monomers like the simulated profile is also located right to the interface. Even though $\mathrm{H}$ monomers are energetically of type A, the fragments with $P$ $<32$ bound to $\mathrm{B}$ chains are now more or less miscible within the $\mathrm{B}$ phase. Although the catalytic $\mathrm{K}$ monomers are not bound and are of great mobility, they nearly and left to the interface become completely consumed. They appear depleted even within the volume B phase indicating an inadequate re-transformation by the reaction of $\mathrm{L}$ monomers. Finally, the calculated curve for the L monomers shows the mobility of this non-bound species and the consumption (overestimated by the model) within the B phase. They rebuild catalytic K monomers, which once again can be incorporated into step (I) of the reaction scheme in Fig. (1b).

As mentioned above, the greatest disagreement between simulated data and the numerical solution of equations (1) is found for the F monomers. Perhaps, the interface reaction step (II) in Fig. (1b) in our model calculation is too fast compared to the reaction steps (I) and (III). To test this assumption, we have altered the activation energy for the three reaction steps in Fig. (1b) systematically and in Fig. (8) we compare the calculated profiles for $\mathrm{C}, \mathrm{D}, \mathrm{F}$ and $\mathrm{H}$ monomers with the original model calculation in the case $E_{A 1}=E_{A 2}=E_{A 3}=0 k_{B} T$.

If we assume as a criterion of acceptance, that the profiles of $\mathrm{C}$ and $\mathrm{D}$ monomers will only be slightly disturbed and the profile for the $\mathrm{F}$ monomers will be distinctly diminished, then we indeed find the calculation in the case $E_{A 1}=0, E_{A 2}=3, E_{A 3}=0 k_{B} T$ to be closer to the simulated data than the other two combinations of activation energies. But to design the equation system (1) more suitable in the prediction of results (without simulations or experiments) of 


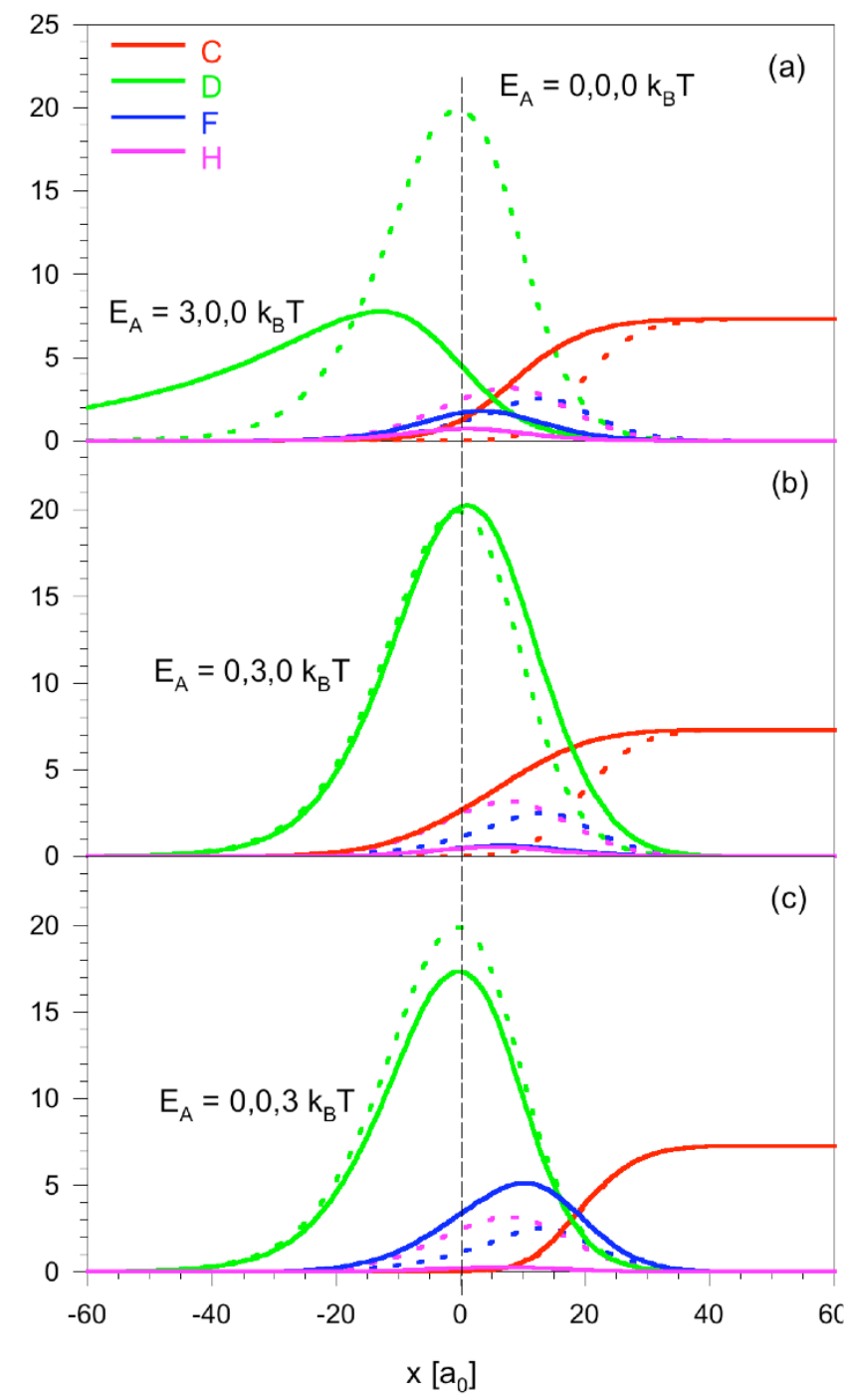

Fig. (8). Calculated concentration profiles for the monomers C, D, $\mathrm{F}$ and $\mathrm{H}$ in the system Split-Kat-A with activation energies $E_{A 1}=E_{A 2}=E_{A 3}=0 k_{B} T$ in model (1) (dashed lines) in comparison with an altered combination of activation energies (solid lines) for steps (I) - (III) in Figure 1b: (a) the combination $(3,0,0)$, (b) the combination $(0,3,0)$ and $(\mathrm{c})$ the combination $(0,0,3) k_{B} T$.

changes in parameters and/or concentrations of reaction participants, the model in a more sophisticated manner should take into account the restricted diffusion at least in the early state of the time evolution. This could be done (but is not yet developed here) by the analysis of the displacement properties of the energetically different species $\mathrm{A} / \mathrm{B}$, which are responsible for the maintenance of the phase separation in the system of immiscible polymers A and B.

\section{ADHESION PROPERTIES}

In order to get a measure for the adhesion properties of the compound on microscopic level, we have adopted a bridge concept coupling tensile strength with the number of connected monomers in $x$ direction and the number of reaction products (copolymers) bridging the interface, respectively. To implement the bridge concept $f_{\text {simple }}$, for each chain, and for each monomer $m_{0}$ in the chain, we count in $x$ direction the number of monomers $m_{L}$ to the left of the monomer $m_{0}$ and the number of monomers $m_{R}$ to the right of the monomer $m_{0}$ and create the product. Additionally, in the concepts $f_{\text {unlike }}$ and $f_{\text {like }}$ we count the number of $r \leq \sqrt{6}$ contacts of $m_{L}, m_{R}$ to monomers of different type in different chains $\left(f_{\text {unlike }}\right)$ und like chains $\left(f_{\text {like }}\right)$ providing a picture of the intra- and intermolecular monomer neighbourhood. For computational intentions this approach in detail reads:

$$
\begin{aligned}
& f_{t y p}(x)=\sum_{i=1}^{N} f_{i}^{L}(x) \cdot f_{i}^{R}(x) \\
& f_{i}^{L}(x)=\sum_{j=1}^{P_{i}} \sum_{k=1}^{P_{i}} p_{i j k}(x) \quad f_{i}^{R}(x)=\sum_{j=1}^{P_{i}} \sum_{k=1}^{P_{i}} q_{i j k}(x) \\
& p_{i j k}(x)=\left\{\begin{array}{ll}
1+\Gamma_{t y p} & \text { for } x_{i k}<x_{i j} \\
0 & \text { for } x_{i k}=x_{i j}
\end{array}\right\} \\
& q_{i j k}(x)=\left\{\begin{array}{ll}
1+\Gamma_{t y p} & \text { for } x_{i k}>x_{i j} \\
0 & \text { for } x_{i k}=x_{i j}
\end{array}\right\} \\
& \Gamma_{t y p}=\left\{\begin{array}{lll}
0 & \text { for typ }=\text { simple } \\
\rho & \text { for typ }=\text { unlike } & \text { number of unlike contacts in } r \leq \sqrt{6} \\
\sigma & \text { for typ }=\text { like } & \text { number of like } \quad \text { contacts in } r \leq \sqrt{6}
\end{array}\right\}
\end{aligned}
$$

with

unlike $=$ contacts of monomers $j, k$ to monomer $m$ of different type in chain $n \neq i$

like $=$ contacts of monomers $j, k$ to monomer $m$ of different type in chain $n=i$

and

$N \quad$... Number of chains

$P_{i} \quad \ldots$ Degree of Polymerization of chain $i$

$p, q \quad \ldots$ Functions of the $x$ position of monomers $j, k$ in chain $i$

$\Gamma_{t y p} \quad \ldots$ Weight additive for contacts depending on type

$\rho, \sigma \quad \ldots$ Counter for weight additive $\Gamma_{t y p}$

which implies, that the evaluation program is capable to identify the objects (single monomers, chains, branched chains etc.) to calculate the neighbourhood of the individual monomers. Apart from the bridging concepts, which partially require considerable computational effort, we have also adopted a very simple, but illustrative concept called the concept of bonding chains. In this concept we count those copolymers, which fulfil the following condition: Let $x_{\min }$ and $x_{\max }$ label the minimum and maximum extension of the copolymer in $x$ direction, then the counter for the concept of bonding chains will increase by 1 when $x_{\min } \leq-R_{G}$ and $x_{\max } \geq+R_{G}$. That means, we look for block copolymers, which have one arm in the corresponding phase A and a second branch in the other phase B, respectively. In this way we have found a chemically interconnecting links between the two immiscible phases, which we believe to enhance the adhesion strength. As a measure for the inter- 
penetrating depth of the molecule into the corresponding phase we have chosen arbitrarily the radius of gyration $R_{G}$. Additionally, we divide this number of chains by the number of reaction events, so that, for convenience, the resulting time dependent function is always $0 \leq f_{\text {bond }} \leq 1$.

In Figs. (9-11) we have compiled the contributions of the two chemical reaction types Split-All and Split-Kat-A to the three local resolved bridge concepts $f_{\text {simple }}, f_{\text {like }}$ and $f_{\text {unlike }}$ at three different simulation times, respectively.

Since the bridge concepts are based on the principle of connectivity, the molecules with higher degree of polymerization than $P=32$ will extensively contribute to the strength enhancement, which surely will be overestimated by the large number of reactive sites in the molecules of type A and $\mathrm{B}$, respectively. However, already the functions for the non-reactive system show some little effects in this context. So the function $f_{\text {unlike }}$ additionally weights the contacts of different monomers in different chains. This is the case in the interface region, which now is marked by a low maximum.

Since the concepts $f_{\text {simple }}$ and $f_{\text {like }}$ without reaction yield the same results, the functions for these concepts are identical for the non-reactive system. The curves exhibit a flat minimum at the mathematical interface and symmetrically to those two small maxima. While the nature of the minimum at the interface may be found in a compression of the chains in $x$ direction (which can be verified calculating the dimension $\Delta x$ of the chains as a function of $x$, not shown here), the origin of the low maxima is determined by the enlarged concentration of chains on both sides of the interface in the non-reacted system. As it was also observed for the concen- tration profiles of reaction products for the catalytic system, the peaks for the bridge functions appear to the right of the mathematical interface and after a certain time they do not grow in height anymore but into the B volume phase. In contrast to this behaviour, the reaction Split-All results in a nearly symmetrical arrangement of the peaks, and with increasing simulation time they grow in height and so also in area.

The reason for this phenomenon may be found in the formation of the large felt-like molecules (discussed above), which for statistical reasons are stretched on both sides of the interface and due to their size are not able to move in $x$ direction anymore. Whereas for the concept $f_{\text {simple }}$ in Fig. (9) without reaction a small depression can be observed at the interface, the reactions strongly increase the strength behaviour to the right of the interdiffussion layer of the two phases (Split-Kat-A) or straight at the interface (Split-All), respectively. Note, however, that the extent of the apparent reinforcement may be strongly overestimated by the large and exaggeratedly number of reactive sites, which is also indicated by the factors in the labels in Fig. (9). As indicated by the arrows, the growth of the function front for the reaction Split-Kat-A occurs into the volume B phase, since reaction products containing only short A chains are now more or less miscible within the B polymers and may penetrate in $x$ direction. The sketch in the upper right corner of the figure qualitatively shows the increase of the peak areas as a measure for the total amount of connections with time. A completely similar behaviour can be observed for the bridge concepts $f_{\text {like }}$ and $f_{\text {unlike }}$, where the near $r \leq \sqrt{6}$ contacts between $\mathrm{A}$ and $\mathrm{B}$ monomers in like $\left(f_{\text {like }}\right)$ and unlike $\left(f_{\text {unlike }}\right)$ molecules

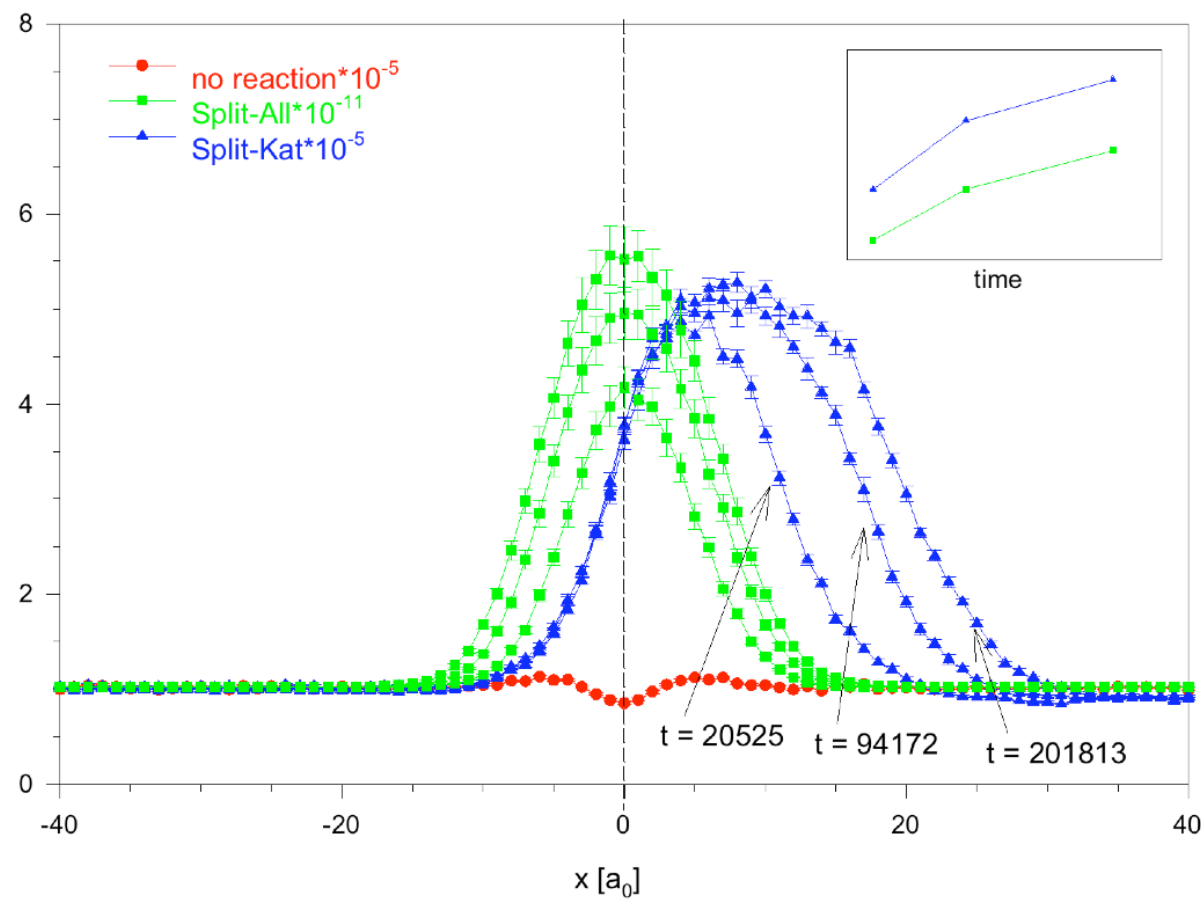

Fig. (9). The bridge concept $f_{\text {simple }}$ for the reactions Split-All and Split-Kat-A at activation energy $E_{A}=0 k_{B} T$ for the three time steps 20525 , 94172 and 201813 MCS compared to the case without reaction. For the reactions, in the upper right corner the progress of the peak areas is shown schematically. 


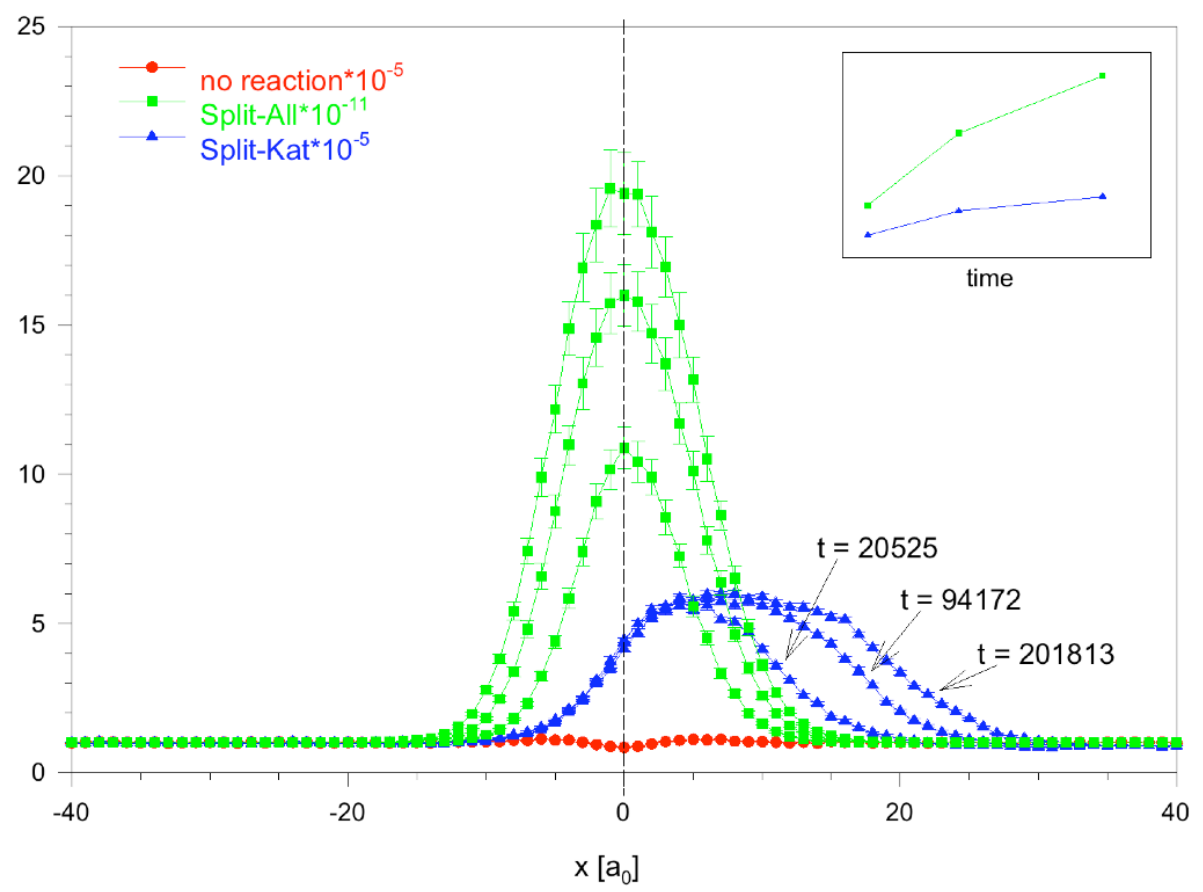

Fig. (10). The bridge concept $f_{\text {like }}$ for the reactions Split-All and Split-Kat-A at activation energy $E_{A}=0 \quad k_{B} T$ for the three time steps 20525 , 94172 and 201813 MCS compared to the case without reaction. For the reactions, in the upper right corner the progress of the peak areas is shown schematically.

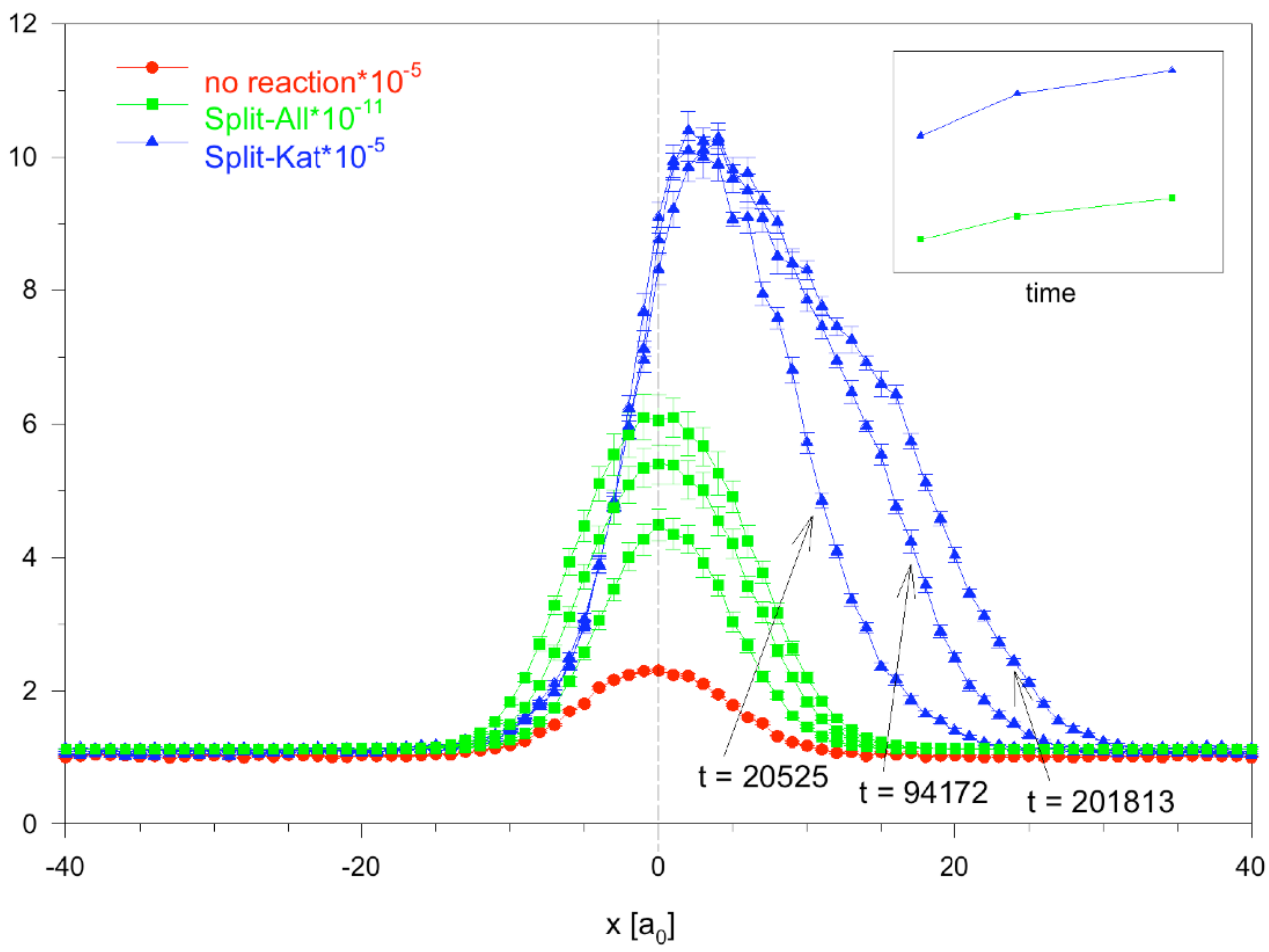

Fig. (11). The bridge concept $f_{\text {unlike }}$ for the reactions Split-All and Split-Kat-A at activation energy $E_{A}=0 \quad k_{B} T$ for the three time steps 20525 , 94172 and 201813 MCS compared to the case without reaction. For the reactions, in the upper right corner the progress of the peak areas is shown schematically.

additionally highlight the interface region and in this way show the adhesive neighbourhood in real polymer systems. Note again, that the function values for both the concepts $f_{\text {like }}$ and $f_{\text {unlike }}$ within the volume phases approach the $f_{\text {simple }}$ limit, since the weight additives for $\mathrm{A} / \mathrm{B}$ contacts in the volume phases are always zero. 


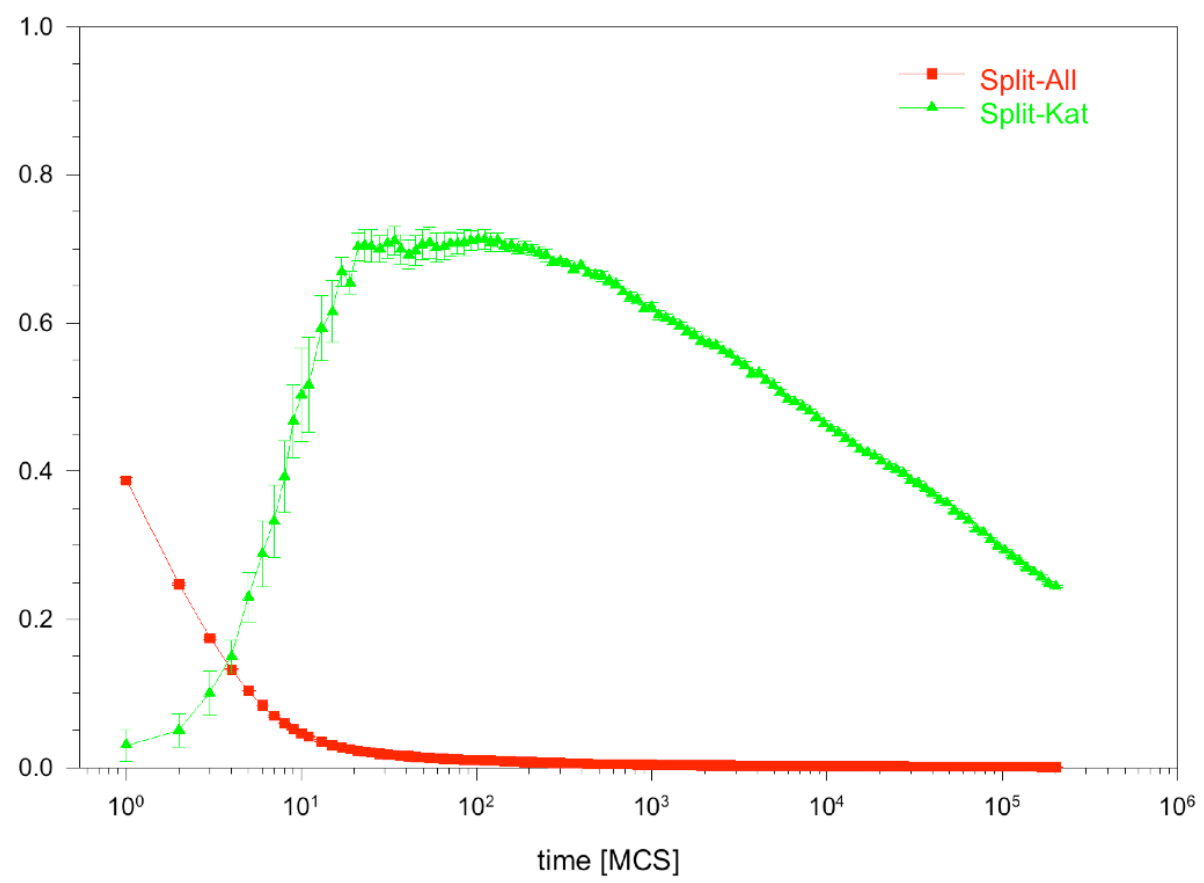

Fig. (12). The results of the concept of bonding chains (bridging chains/reacted monomers) for the reactions Split-All and Split-Kat-A at activation energy $E_{A}=0 \quad k_{B} T$ as a function of the simulation time.

The main disadvantage of the three bridge concepts is the incapability to explain the relative strength between interface and volume part of the compound. According to these concepts the fracture should occur in the volume, but not near the interface, which is in most cases far away from practical observations.

The concept of bonding chains (Fig. 12) is not locally resolved in a direct way, but mediately, because it counts only those copolymers crossing the mathematical interface having one arm within the A volume and the other branch within the B phase.

This very illustrative concept, especially for very short times, shows the benefit of the reaction Split-All in creating embedded block copolymers in the very early state of the simulation, which seems favourable in the framework of the two-component injection molding. Although the number of bridging chains for the reaction Split-Kat-A is nearly twice as high as for the reaction Split-All, their effect is delayed by the lower rate of the preceding reaction. The rapid decay of the curve for the reaction Split-All is caused by the ratio shape of the function value, where the denominator (the consumption) always increases, but the numerator (the number of bridging chains) is limited because of space reasons due to the saturation of the interface region by reaction products. However, it remains an open question, to what extent the two reaction types really will contribute to the adhesion strength - one effect is faster, the other effect is higher in function value, but is acting later in time.

\section{COMPARISON OF REACTIONS}

Finally, as mentioned above in the text, the step (II) of the reaction Split-Kat-A in principle is the same as in the reaction Split-Mid, which we have investigated in earlier work [28]. This step (see Fig. 1b) consists in the attack of the reactive monomer $\mathrm{C}$ at the centre of a $\mathrm{B}$ chain on a arbitrary monomer of the A chain resulting in a splitting of $\mathrm{A}$ and the formation of a unsymmetrical diblock copolymer in conjunction with a fragment of the A chain. In Fig. (13a) we have compiled the consumption curves of the reaction Split-Mid for the activation energies $E_{A}=0,1,3$ and $5 k_{B} T$ compared to the corresponding function for the reaction Split-Kat-A at $E_{A}=0 k_{B} T$.

Due to the prefixed activation step (I) the catalytic reaction is slowed down being now comparable with the rate of the reaction Split-Mid at $E_{A}=3 k_{B} T$. So, for example, a consumption of 10 units of reactive $\mathrm{C}$ monomers (reaction events) in the reaction Split-Mid at $E_{A}=0 k_{B} T$ is obtained already after $t_{M i d 1} \approx 2 M C S$ and at $E_{A}=3 k_{B} T$ after $t_{\text {Mid } 2} \approx 48 M C S$, while the same consumption in the reaction Split-Kat-A at $E_{A}=0 k_{B} T$ is achieved not before a time $t_{K a t} \approx 80 M C S$. But it still remains the question, which consequences for the strength behaviour arise from these differences in the consumption as a function of time. As it is shown in Fig. (13b), at $E_{A}=0 k_{B} T$ after termination of the simulation at time $t=201813$ MCS, the bridge concepts $f_{\text {simple }}$ and $f_{\text {like }}$ indicate the slightly better performance for the reaction Split-Mid, but the concept $f_{\text {unlike }}$ weighting contacts of different monomers in different chains with more impact clearly favours the reaction Split-Kat-A in spite of their lower reaction rate. 


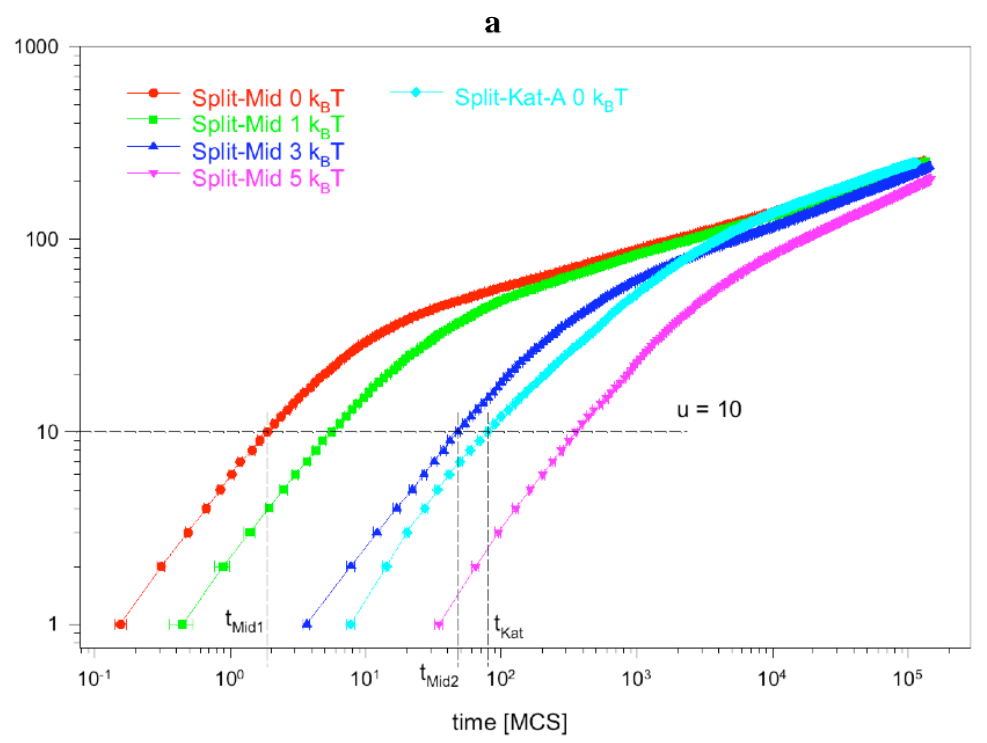

b
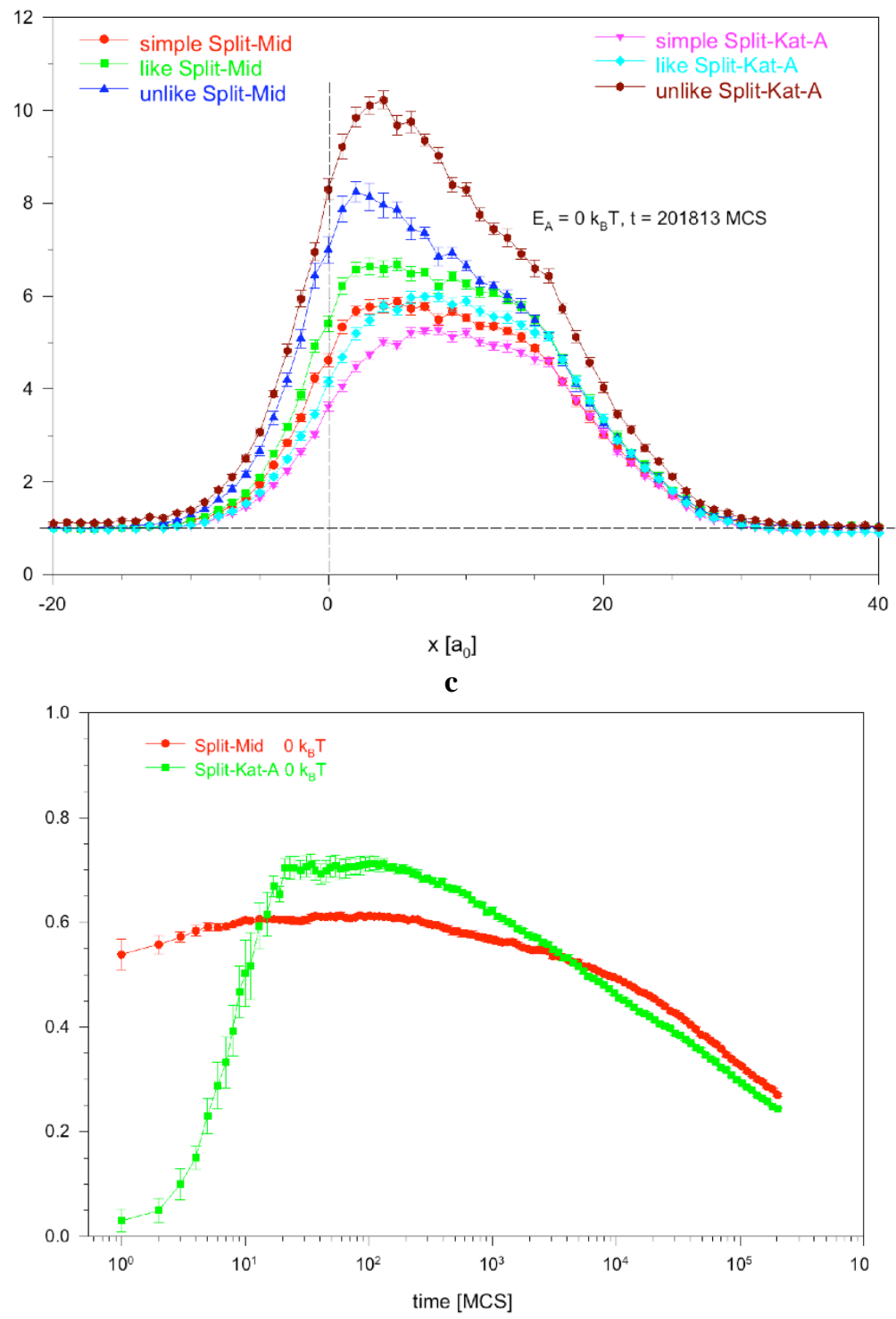

Fig. (13). Comparison between simulation results of the previously discussed reaction Split-Mid [28] and the reaction Split-Kat-A: (a) the consumption curves for Split-Mid at $E_{A}=0,1,3$ and $5 k_{B} T$ and for Split-Kat-A at $E_{A}=0 k_{B} T$, (b) the bridge concepts $f_{\text {simple }}, f_{\text {like }}$ and $f_{\text {unlike }}$ for $E_{A}=0 k_{B} T$ at time $t=201813 M C S$ and (c) the concept of bonding chains for $E_{A}=0 k_{B} T$. 
This effect probably may be attributed to the fast activation of $\mathrm{G}$ monomers forming reactive $\mathrm{D}$ by mobile free $\mathrm{K}$ monomers up to the volume of the A phase, which is also visible in Fig. (6) for the concentration profile of the D monomers. Since the total building rate of reaction products is delayed, transport phenomena can create new space for the formation of intermolecular monomer contacts, which are visible in the function $f_{\text {unlike. }}$. In Fig. (13c) we have compared the curves for the concept of bonding chains (number of bridging copolymers (see text above) per number of reaction events) for the reaction Split-Mid and Split-Kat-A at $E_{A}=0 k_{B} T$ as a function of time.

Even though the latter reaction exhibits a lower reaction rate, the relative number of connecting (strength interfering) block copolymers at times $t \approx 10-1000 M C S$ being essential for the success of the two-component injection molding, exceeds the corresponding relative number of chains for the reaction Split-Mid. This effect strongly indicates the usefulness of a catalytic reaction mechanism compared to a simple interface reaction for the enhancement of the adhesion strength of the compound, since the mechanism can increase the efficiency of the acting reaction events.

\section{CONCLUSIONS}

In the present Monte-Carlo simulation study, which is based on the three dimensional coarse-grained Bond Fluctuation Model, we qualitatively characterized the behaviour of chemical reactions of the Split type being of importance for the reactive compatibilization in two-component injection molding technique. All irreversible reactions are governed by activation energies $E_{A}=0,1,3$ and $5 k_{B} T$, i.e. values which successively slowing down the reaction speed but do not alter the reaction behaviour qualitatively. The reactions result in a characteristic distribution of the degree of polymerization and the reaction products (the concentration profiles) within the simulation volume. The effects found for a specific bridging concept and the concept of bonding chains strongly indicate also the usefulness of a catalytic reaction mechanism for the enhancement of the adhesion strength of the compound, since compared to the corresponding simple mechanism a higher efficiency of the reacting events can be achieved. Compiling the results for all the bridge concepts and the concept of the bonding chains, the reaction Split-All exhibiting as many as possible reactive sites should lead to the most suitable behaviour reinforcing the interface of the two immiscible polymer melts and therefore should be the preferred reaction for compatibilization efforts in the twocomponent injection molding technique.

\section{ACKNOWLEDGEMENTS}

Financial support from Deutsche Forschungsgemeinschaft (DFG) is acknowledged. We thank Dr. Michael Lang from the research group Theory of Polymers at the Leibniz Institut für Polymerforschung Dresden e. V. for helpful discussions.

\section{APPENDIX}

[9]

\begin{tabular}{|c|c|c|c|}
\hline \multirow[t]{4}{*}{$P_{3}^{0}$} & - & 1.80272701280 & $\mathrm{e}+0$ \\
\hline & - & 3.12512929000 & $\mathrm{e}-2$ \\
\hline & - & 1.51788895500 & e-1 \\
\hline & + & 4.46897659000 & $\mathrm{e}-2$ \\
\hline \multirow[t]{6}{*}{$P_{5}^{0}$} & - & 6.35444383634 & $\mathrm{e}+1$ \\
\hline & + & 8.44423032590 & $\mathrm{e}+1$ \\
\hline & - & 4.57655164532 & $\mathrm{e}+1$ \\
\hline & + & 1.21845931281 & $\mathrm{e}+1$ \\
\hline & - & 1.58833401390 & $\mathrm{e}+0$ \\
\hline & + & 8.10736776000 & $\mathrm{e}-2$ \\
\hline \multirow[t]{3}{*}{$P_{2}^{0 A}$} & + & 7.31533119630 & e-3 \\
\hline & + & 4.65037615080 & e-7 \\
\hline & - & 2.04196064220 & $\mathrm{e}-12$ \\
\hline \multirow[t]{4}{*}{$P_{3}^{2}$} & - & 1.67336687400 & $\mathrm{e}+0$ \\
\hline & - & 1.58089796000 & e-1 \\
\hline & - & 1.13285514000 & $\mathrm{e}-1$ \\
\hline & + & 1.84147777000 & $\mathrm{e}-2$ \\
\hline \multirow[t]{3}{*}{$P_{2}^{2}$} & - & 1.06209641290 & $\mathrm{e}+0$ \\
\hline & - & 6.03243787700 & e-1 \\
\hline & + & 1.77446225000 & $\mathrm{e}-2$ \\
\hline \multirow[t]{2}{*}{$P_{1}^{2 E}$} & + & 9.24776547150 & $\mathrm{e}+0$ \\
\hline & + & 5.45218273840 & $e-5$ \\
\hline \multirow[t]{2}{*}{$P_{1}^{2 A}$} & + & 7.07522345280 & $e-5$ \\
\hline & - & 5.45218273840 & e-11 \\
\hline
\end{tabular}

\section{REFERENCES}

[1] Utracki, L. Polymer Alloys and Blends, Carl Hanser: New York, 1989.

[2] Wool, R.P. Polymer Interfaces: Structure and Strength, Carl Hanser: New York, 1995.

[3] Macosko, C.W.; Jeon, H.K.; Hoye, T.R. Reactions at polymerpolymer interfaces for blend compatibilization. Prog. Polym. Sci., 2005, 30(8-9), 939-947.

[4] Bates, F.S.; Fredrickson, G.H. Block Copolymer Thermodynamics - Theory and Experiment. Annu. Rev. Phys. Chem., 1990, 41, 525557.

[5] Binder, K. Phase-Transitions in Polymer Blends and BlockCopolymer Melts - Some Recent Developments. Adv. Polym. Sci., 1994, 112, 181-299.

[6] Anastasiadis, S.H.; Gancarz, I.; Koberstein, J.T. Compatibilizing Effect of Block Copolymers Added to the Polymer-Polymer Interface. Macromolecules, 1989, 22(3), 1449-1453.

[7] Brown, S.B. Chemical Processes Applied to Reactive Extrusion of Polymers. Annu. Rev. Mater. Sci., 1991, 21, 409-435.

[8] Nishio, T.; Suzuki, Y.; Kojima, K.; Kakugo, M.J. Morphology of Maleic-anhydride Grafted Polypropylene and Polyamide Alloy Produced by Reactive Processing. Polym. Eng., 1991, 10(1-3), 123149.

Coefficients of the polynoms
Guegan, P.; Macosko, C.W.; Ishizone, T.; Hirao, A.; Nakahama, S. Kinetics of Chain Coupling at Melt Interfaces. Macromolecules, 1994, 27(18), 4993-4997. 
[10] Nagel, J.; Bräuer, M; Hupfer, B.; Grundke, K.; Schwarz, S.; Lehmann, D. Investigations on the Reactive Surface Modification of Polycarbonate by Surface-Reactive Injection Molding. J. Appl. Polym. Sci., 2004, 93(3), 1186-1191.

[11] Yin, Z.; Koulic, C.; Pagnoulle, C.; Jerome, R. Probing of the Reaction Progress at a PMMA/PS Interface by Using AnthraceneLabeled Reactive PS Chains. Langmuir, 2003, 19(2), 453-457.

[12] Jeon, H.K.; Macosko, C.W.; Moon, B.; Hoye, T.R.; Yin, Z. Coupling Reactions of End- vs Mid Functional Polymers. Macromolecules, 2004, 37(7), 2563-2571.

[13] Jones, T.D.; Schulze, J.S.; Macosko, C.W.; Lodge, T.P. Effect of Thermodynamic Interactions on Reactions at Polymer/Polymer Interfaces. Macromolecules, 2003, 36(19), 7212-7219.

[14] Nagel, J.; Scheidler, D.; Hupfer, B.; Bräuer, M.; Pleul, D.; Vogel, C.; Lehmann, D.; Amesöder, S.; Investigations on the Formation of Composites by Injection Molding of PA6 and Different Grafted Polypropylenes and their Blends. J. Appl. Polym. Sci., 2006, 100(4), 2992-2999.

[15] Fredrickson, G.H. Diffusion-controlled Reactions at PolymerPolymer Interfaces. Phys. Rev. Lett., 1996, 76(18), 3440-3443.

[16] O'Shaughnessy, B.; Sawhney, U. Polymer Reaction Kinetics at Interfaces. Phys. Rev. Lett. 1996, 76(18), 3444-3447.

[17] Fredrickson, G.H.; Milner, S.T. Time-Dependent Reactive Coupling at Polymer-Polymer Interfaces. Macromolecules, 1996, 29(23), 7386-7390.

[18] O' Shaughnessy, B.; Vavylonis, D. Reactions at Polymer Interfaces: Transitions from Chemical to Diffusion-Control and Mixed order Kinetics. Europhys. Lett., 1999, 45(5), 638-644.

[19] Müller, M. Reactions at Polymer Interfaces: A Monte-Carlo Simulation. Macromolecules, 1997, 30(20), 6353-6357.

[20] Yeung, C.; Herrmann, K.A. Molecular Dynamics Simulation of Reactive Compatibilization of Polymer Blends. Macromolecules, 2003, 36(1), 229-237.

[21] He, X.; Nagel, J.; Lehmann, D.; Heinrich, G. Interface Structure Between Immiscible Reactive Polymers Under Transreaction: a Monte-Carlo Simulation. Macromol. Theory Simul., 2005, 14(5), 305-311.

[22] Carmesin, I.; Kremer, K. The Bond Fluctuation Method - a New Effective Algorithm for the Dynamics of Polymers in All Spatial Dimensions. Macromolecules, 1988, 21 (9), 2819-2823.
[23] Deutsch, H.P.; Binder, K. Interdiffusion and Self-Diffusion in Polymer Mixtures - a Monte-Carlo Study. J. Chem. Phys., 1991, 94(3), 2294-2304.

[24] Deutsch, H.P.; Binder, K. Critical-Behavior and Crossover Scaling in Symmetrical Polymer Mixtures - a Monte-Carlo Investigation. Macromolecules, 1992, 25(23), 6214-6230.

[25] Müller, M.; Miscibility Behavior and Single Chain Properties in Polymer Blends: a Bond Fluctuation Model Study. Macromol. Theory Simul. 1999, 8(4), 343-374.

[26] John, A.; Nagel, J.; Heinrich, G. Monte-Carlo Simulation of Polymer Reactions at Interfaces. Macromol. Theory Simul., 2007, 16(4), 430-440.

[27] John, A.; Sommer, J.U. Monte-Carlo Simulation of the Reactive Formation of Co-Continuous Nanostructured Polymers. Macromol. Theory Simul., 2008, 17(6), 274-279.

[28] John, A., Nagel, J., Heinrich, G.; Compatibilization in TwoComponent Injection Molding by Means of Split Reactions with Varying Reactive Sites - a Monte-Carlo Simulation. Open Macromol J 2011; 5: 1-12.

[29] Fakirov, S; Sarkissova, M; Denchev, Z. Sequential Reordering in Condensation Copolymers. 1. Melting and Crystallization-Induced Sequential Reordering in Immiscible Blends of Poly(ethylene terephthalate) with Polycarbonate or Polyarylate. Macromol. Chem. Phys, 1996, 197(9), 2837-2867.

[30] Metropolis, N.; Rosenbluth, A.N.; Rosenbluth, M.N.; Teller, A.H.; Teller, E.; Equation of State Calculations by Fast Computing Machines. J. Chem. Phys. 1953, 2l(6), 1087-1092.

[31] Kreer, T.; Baschnagel, J.; Müller, M.; Binder, K. Monte-Carlo Simulation of Long Chain Polymer Melts: Crossover from Rouse to Reptation Dynamics. Macromolecules, 2001, 34(4), 1105-1117.

[32] Doi, M.; Edwards, S.F.; The Theory of Polymer Dynamics, Clarendon Press: Oxford, 2004.

[33] Islam, M.A.; Einstein-Smoluchowski Diffusion Equation: a Discussion. Physica Scripta, 2004, 70(2-3), 120-125.

[34] Schwarz, H.R.; Köckler, N.; Numerische Mathematik, Teubner: Wiesbaden, 2004.

Received: August 30, 2011

Revised: November 28, 2011

Accepted: November 30, 2011

(C) John et al.; Licensee Bentham Open.

This is an open access article licensed under the terms of the Creative Commons Attribution Non-Commercial License (http://creativecommons.org/licenses/by-nc/3.0/) which permits unrestricted, non-commercial use, distribution and reproduction in any medium, provided the work is properly cited. 